\title{
Recent progress of particle migration in viscoelastic fluid
}

Dan Yuan', Qianbin Zhao ${ }^{a}$, Sheng Yan", Shi-Yang Tang ${ }^{a}$, Gursel Alici ${ }^{a}$, Jun Zhang ${ }^{\text {b,* }}$ and Weihua $\mathrm{Li}^{\mathrm{a}}$,*

${ }^{a}$ School of Mechanical, Materials and Mechatronic Engineering, University of Wollongong, Wollongong, NSW 2522, Australia

${ }^{b}$ School of Mechanical Engineering, Nanjing University of Science and Technology, Nanjing 210094, China

*Corresponding authors: weihuali@uow.edu.au.

junzhang@njust.edu.cn. 


\begin{abstract}
Recently, research on particle migration in non-Newtonian viscoelastic fluids has gained considerable attention. In a viscoelastic fluid, three dimensional (3D) particle focusing can be easily realized in simple channels without the need of any external force fields or complex microchannel structure compared with that in a Newtonian fluid. Due to its promising property on particle precise focusing and manipulation, this field has been developed rapidly, and the research on the field has been shifted from fundamentals to applications. This review will elaborate the recent progress of particle migration in viscoelastic fluids, especially on the aspect of applications. The hydrodynamic forces on the micro/nano particles in viscoelastic fluids are discussed. Next, we elaborate the basic particle migration in viscoelasticity-dominant fluid and elasto-inertial fluid in straight channels. After that, a comprehensive review on the applications of viscoelasticity-induced particle migration (particle separation, cell deformability measurement and alignment, particle solution exchange, Rheometry-on-a-chip and others) is presented; Finally, we thrash out some perspectives on the future directions of particle migration in viscoelastic fluids.
\end{abstract}

Keywords: Microfluidics, Viscoelastic fluid, Particle migration, Particle focusing, Particle separation 


\section{Introduction}

Microfluidics, which can precisely manipulate and control fluids and particles at micron and submicron dimensions, has many advantages compared with the traditional macroscale platform, including reduced sample volumes, low cost, fast processing and analysing, high efficiency, high sensitivity, and high accuracy ${ }^{1,2}$. Currently, it has been widely used in medical, chemical, biological and environmental fields ${ }^{3-5}$. Particle manipulating technologies such as focusing ${ }^{6,7}$, separation ${ }^{8}$, trapping ${ }^{9}$, and stretching ${ }^{10,11}$, are indispensable abilities in microfluidics. A variety of particle manipulating technologies have been developed based on either active approaches using external forces induced by electric ${ }^{12-15}$, magnetic 16, 17, acoustic $^{18-20}$ and optical ${ }^{21}$ fields; or passive intrinsic hydrodynamic forces ${ }^{6,22-28}$. However, most of these manipulating methods are performed and studied in Newtonian fluids. In fact, non-Newtonian fluids such as blood, cytoplasm, and many other body fluids, are very ubiquitous in our daily life and in real world issues. Therefore, it is important to investigate particle migration in non-Newtonian fluids to develop deep understanding of cell behaviours in these body fluids. A non-Newtonian fluid is a fluid that does not follow Newton's Law of Viscosity. Newton's law of viscosity defines the relationship between the shear stress and shear rate of a fluid subjected to a mechanical stress. The non-Newtonian fluid either has a shear-dependent or shear-independent viscosity. The non-Newtonian fluid can be formed by dissolving long chain molecules (PEO (poly (ethylene oxide), PVP (poly (vinyl pyrrolidone), PAA (polyacrylamide), PAM (polyacrylamide), DNA, HA (hyaluronic acid)) in Newtonian base medium. The polymers can induce viscoelastic effect in their dissolved solutions.

Initially, particle migration in a viscoelastic non-Newtonian fluid was investigated in a channel with a macroscale dimension. These studies were generally conducted using two typical flows: Poiseuille flow in a circular pipe and Couette flow in two parallel plates. In 1966, Karnis and Mason first studied the migration of a sphere in a viscoelastic liquid in a 
pipe flow (pipe diameter $\sim \mathrm{cm}$ ) when inertia is negligible, and observed the sphere approaches the centre of the pipe regardless of its initial position ${ }^{29}$. After that, the motion of particles in Couette flows (the laminar flow of a viscous fluid in the space between two parallel plates, of which one is moving relative to the other) was studied experimentally ${ }^{30,31}$. Trhrani ${ }^{32}$ investigated experimentally on particle migration in viscoelastic fluids used in hydraulic fracturing. He found that the migration occurs toward the region of lower shear rate in the suspensions with a moderate particle volume fraction. Thus in Boger fluid ${ }^{33}$, an elastic fluid with constant viscosity which is independent of the shear rate, particles are pushed towards the centreline in case of Poiseuille flow (steady viscous fluid flow driven by an effective pressure gradient established between the two ends of a long straight pipe with a uniform circular cross-section) while aggregate close to the outer wall in a Couette flow. Ho and Leal ${ }^{34}$ theoretically studied the lateral migration of a neutrally buoyant rigid sphere suspended in a second order fluid and demonstrated that the particle migration is induced by the non-uniform normal stress distribution and particles move towards the low normal stress difference regions. Later, Huang et al. ${ }^{35}$ conducted a direct simulation of the motion of solid particles in Couette and Poiseuille flows of viscoelastic fluids, and found inertia, elasticity, shear thinning and the blockage ratio of the channel affect the stable equilibrium positions of neutrally buoyant particles. Moreover, through the same method, Huang and Joseph ${ }^{36}$ specifically studied the effects of shear thinning on migration of neutrally buoyant particles in a pressure driven flow. As appose to the fact that the inertia tends to push the particles away from both the walls and the centerline in Newtonian fluids, in viscoelastic fluids with shearthinning effects, an annular particle-free zone at an intermediate radii can be created due to the fact that the elasticity causes them to migrate toward the centreline, and shear thinning moves the particles away from the centerline but toward the channel walls. The annular particle-free zone expands with the increment of shear thinning. 
Recently, due to the advancement of micro- and nano-fabrication technology and blooming of microfluidics, researchers started to investigate particle behaviours in viscoelastic fluids in a confined channel with a micrometer scale. Based on $3 \mathrm{D}$ numerical simulations and experiments, D' Avino ${ }^{37}$ demonstrated the presence of a bistability scenario for transversal migration of particles suspended in a viscoelastic fluid in a micro-pipe. They observed a neutral cylindrical surface, which acts as a separatrix of particles' initial positions and divides particles to migrate either to the channel wall or the centreline.

The recent increasing attention on particle migration based on viscoelastic fluid is a result of its interesting intrinsic fluid properties. Compared with particle focusing in a Newtonian fluid, in a viscoelastic fluid, 3D particle focusing can be easily realized in simple channels without any external force fields or complex microchannel structure, and for particles with a much wider size range (from submicrometer to even nanometer). These advantages of viscoelastic fluids allow fast growth of microfluidic applications based on viscoelastisity-induced particle migration. Recent review articles from D'Avino et al. ${ }^{38}$ and Lu et al. ${ }^{39}$ described the mechanism of viscoelasticity-driven particle migration from both experiments and theoretical analysis, and various passive particle manipulation techniques, respectively. Given that the fundamentals involving viscoelastic fluids have been extensively studied, and investigation of viscoelastic fluids for biomedical and chemical applications within the context of microfluidics has recently gained momentum and is evolving very quickly, in this review, we discuss the up-to-date progress of particle migration in viscoelastic fluids mainly from the aspect of applications, laying out a comprehensive perspective on their potentials in future lab-on-a-chip platforms. In addition, we propose several potential future research directions that probably deserve more attention and efforts from the microfluidic community. This paper is organized as follows. Section 2 will introduce hydrodynamic forces in viscoelastic fluids. The basic particle migration in 
viscoelasticity-dominant fluid and elasto-inertial fluid in straight channels will be introduced in section 3. In section 4, we will comprehensively discuss the applications of viscoelasticityinduced particle migration in microfluidics, including particle separation (with the aid of sheath flow; sheathless particle separation in symmetric bifurcation channel, with the aid of dean flow, and with the aid of magnetophoresis), cell deformability measurement and alignment, particle solution exchange, Rheometry-on-a-chip and other applications developed in microfluidics (mixing, heat transfer, droplet generation and sorting, and so on). Finally, the perspectives on the future directions of conducting particle migration in viscoelastic fluid will be discussed in section 5. The overview of this review is shown in Figure 1.

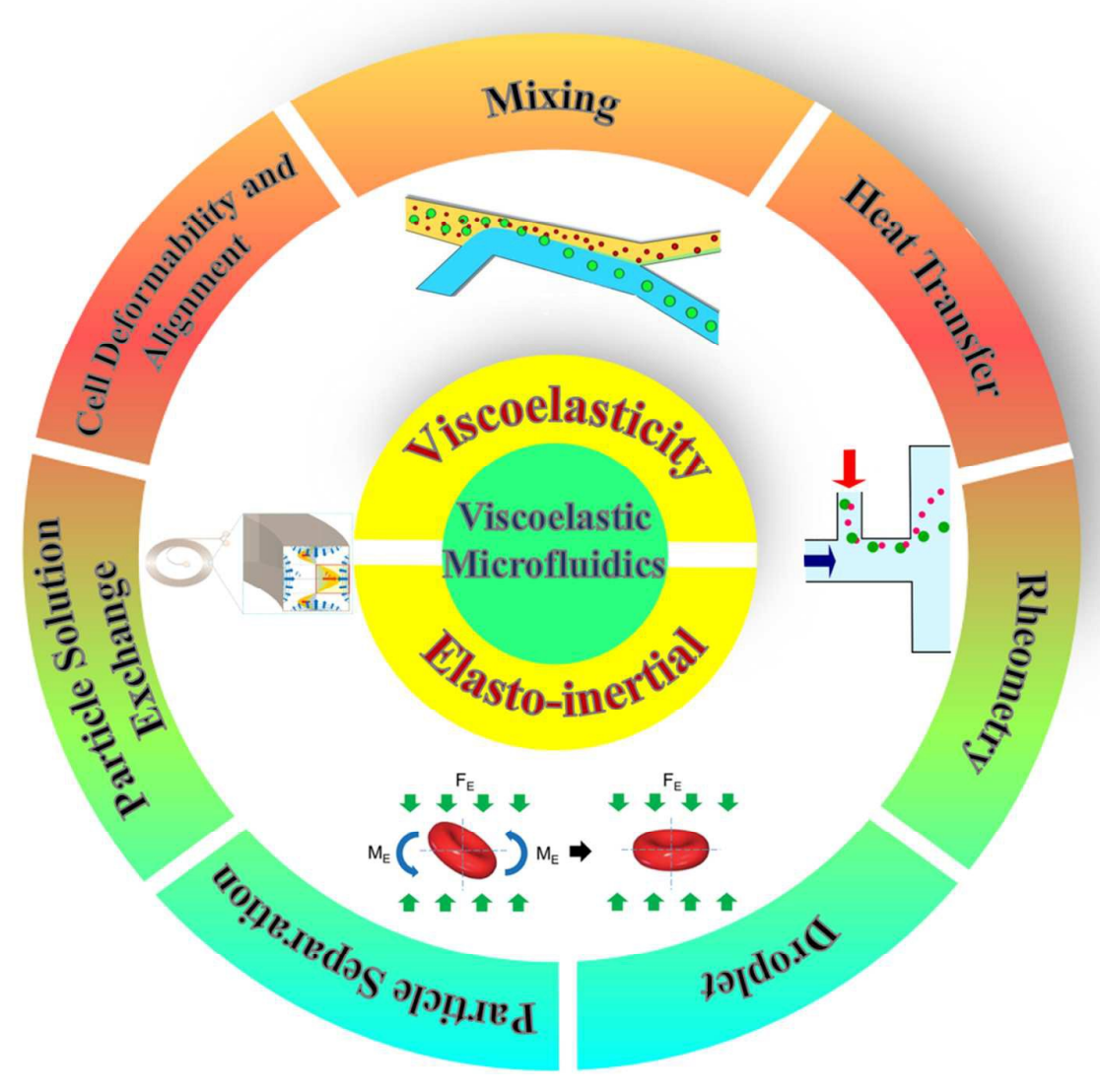

Figure 1 Overview of particle migration in viscoelastic fluids. 


\section{Hydrodynamic forces in viscoelastic fluids}

In viscoelastic fluid, both the inertial effect and viscoelastic effect may contribute to particle migration. Three hydrodynamic forces may exert on particles flowing in viscoelastic fluids: elastic force $F_{E}$, inertial lift force $F_{L}$, and drag force $F_{D}$. Consequently, the particle trajectories and equilibrium positions are tuned by competition of these forces. A schematic of the forces exerting on particles in viscoelastic fluids is shown in Figure 2.

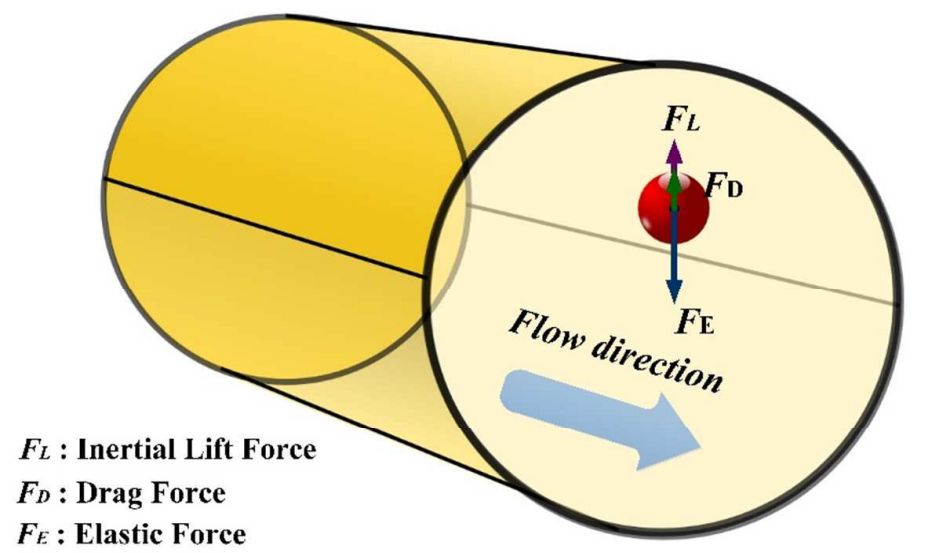

Figure 2 Schematic of the forces exerting on particles in viscoelastic fluids.

\subsection{Inertial lift force}

When particles are flowing in a fluid with un-negligible inertia, they are affected by an inertial lift force. The inertial effect has been used for various microfluidic applications such as focusing ${ }^{40,41}$, separation ${ }^{42,43}$, and filtration ${ }^{44,45}$. The inertial lift force $F_{L}$, which is comprised of the shear gradient lift force and wall lift force, induces the lateral migration of particles toward equilibrium positions between channel centerline and channel walls. The inertial lift force can be expressed as ${ }^{6,46}$ :

$$
\begin{gathered}
F_{L}=\frac{\rho_{f} V_{m}^{2} a^{4}}{D_{h}^{2}} f_{L}\left(R_{c}, x_{c}\right) \\
R_{C}=\frac{\rho_{f} V_{m} D_{h}}{\mu_{f}}
\end{gathered}
$$


where $\rho_{f}$ is the fluid density. $\mu_{f}$ is the dynamic viscosity; $D_{h}$ is the hydraulic diameter for a channel. $V_{m}$ is the average velocity of the channel flow. $a$ is the particle diameter. $f_{L}\left(R_{c}, x_{c}\right)$ is the lift coefficient of net inertial lift force ${ }^{6} . R_{c}$ is the Reynolds number.

\subsection{Elastic force}

When particles are flowing in a viscoelastic fluid, they are affected by an elastic force. The Weissenberg number $W_{i}$ (or Deborah number $D_{\mathrm{e}}$ ), which is the ratio of the relaxation time $\lambda$ of the fluid to the characteristic time $t_{f}$ of the channel flow, is a dimensionless number to characterise the elastic effects ${ }^{47}$ :

$$
W_{i}=\frac{\lambda}{t_{f}}=\lambda \dot{\gamma}=\lambda \frac{2 V_{m}}{D_{h}}
$$

where $\dot{\gamma}$ is the average (characteristic) shear rate, and can be expressed as $2 V_{m} / D_{h}$. In viscoelastic fluid, both the first $\left(N_{l}=\tau_{x x}-\tau_{y y}\right)$ and second normal stresses $\left(N_{2}=\tau_{y y}-\tau_{z z}\right)$ contribute to particle migration. $\tau_{x x}, \tau_{y y}$, and $\tau_{z z}$ are normal stresses that direct toward the flow, the velocity gradient and vorticity direction, respectively. Normally, the effects of $\mathrm{N}_{2}$ can be neglected in the diluted viscoelastic solutions, because $N_{l}$ is much larger than $N_{2}{ }^{48,49}$. When the $W i$ is very small, the elastic force $F_{E}$ originates from an imbalance in the distribution of $N_{l}$ over the size of the particle ${ }^{39}$ can be expressed as:

$$
F_{E}=C_{e L} a^{3} \nabla N_{1}=C_{e L} a^{3}\left(\nabla \tau_{x x}-\nabla \tau_{y y}\right)=-2 C_{e L} a^{3} \eta_{p} \lambda \nabla \dot{\gamma}^{2}
$$

where $C_{e L}$ is the non-dimensional elastic lift coefficient, $\eta_{p}$ is the polymeric contribution to the solution viscosity.

The relative importance of elastic to inertial effects can be defined as $E l$, which results in different particle migration behaviours in viscoelastic fluids. $E l$ is expressed as the ratio of Weissenberg number $W_{i}$ and Reynolds number $R_{c}$ : 


$$
E l=\frac{W_{i}}{R_{c}}=\frac{2 \lambda \mu_{f}}{\rho_{f} D_{h}^{2}}
$$

\subsection{Drag force}

The viscous drag force, which is induced due to the velocity difference of fluid element and particles, can also affect particle migration. Assuming a spherical particle travelling in a uniform Stokes flow, the drag force can be expressed as ${ }^{6,28}$ :

$$
F_{D}=3 \pi \mu_{f} a\left(v_{f}-v_{p}\right)
$$

where $v_{f}$ and $v_{p}$ represent the velocities of fluid elements and particles, respectively. 


\section{Basic particle migration in viscoelastic fluids in straight channels}

In viscoelastic fluid, the relative importance of elastic effect to inertial effect leads to different particle migration phenomenon. In the following sections, we'll describe the basic particle migration in (i) viscoelasticity-dominant flow, and (ii) in elasto-inertial flow.

\subsection{Particle migration in viscoelasticity-dominant flow}

If particles migrate in viscoelastic fluid flow with negligible inertia, it's called the viscoelasticity-dominant particle migration.

Numerical simulations and experiments were employed to study viscoelasticityinduced focusing of particles in pressure-driven wide slit ${ }^{50}$, circular ${ }^{51}$ and square-shaped microchannel ${ }^{52-54}$ under inertialess conditions.

In general, in elasticity dominant flow with inertia neglected, a separatrix divided the particles migration direction according to particles initial positions, Figure 2(a-c). Particles migrate toward centerline if they are inside the separatrix, and migrate towards the wall/corner if they are outside of the separatrix. The separatrix can be regarded as the locus of particle positions where migration velocity of particles is zero. However, these positions are unstable positions, because the net forces on particles along both sides are directing outward, and a slight disturbance may push particles toward the close walls or the centerline. Besides, the position of the separatrix depends on rheological properties of the suspending fluid. The separatrix moves towards the wall as the shear-thinning become less pronounced. A weaker shear-thinning can also slow down the lateral migration of particles. Meanwhile, increasing De (or Wi), the separatrix moves towards the channel centerline, thus increasing the cornerattractive region ${ }^{55}$.

In Giesekus fluid (viscoelastic fluid based on Giesekus model), due to the existence of a secondary normal stress difference, secondary flows which is orthogonal to the flow direction appears, Figure 3(d). The existence of secondary flows strongly affects the particle 
migration dynamics, giving rise to complex behaviors depending on the blockage ratio and the Deborah number. For a sufficiently small particle size and relatively large Deborah number, the velocity components of the secondary flows overcome the migration velocity, and new attractor appears to drive particles to follow vortex trajectories. On the other hand, as the Deborah number decreases and particle diameter increases, the attractor will disappear 55 .

(a)
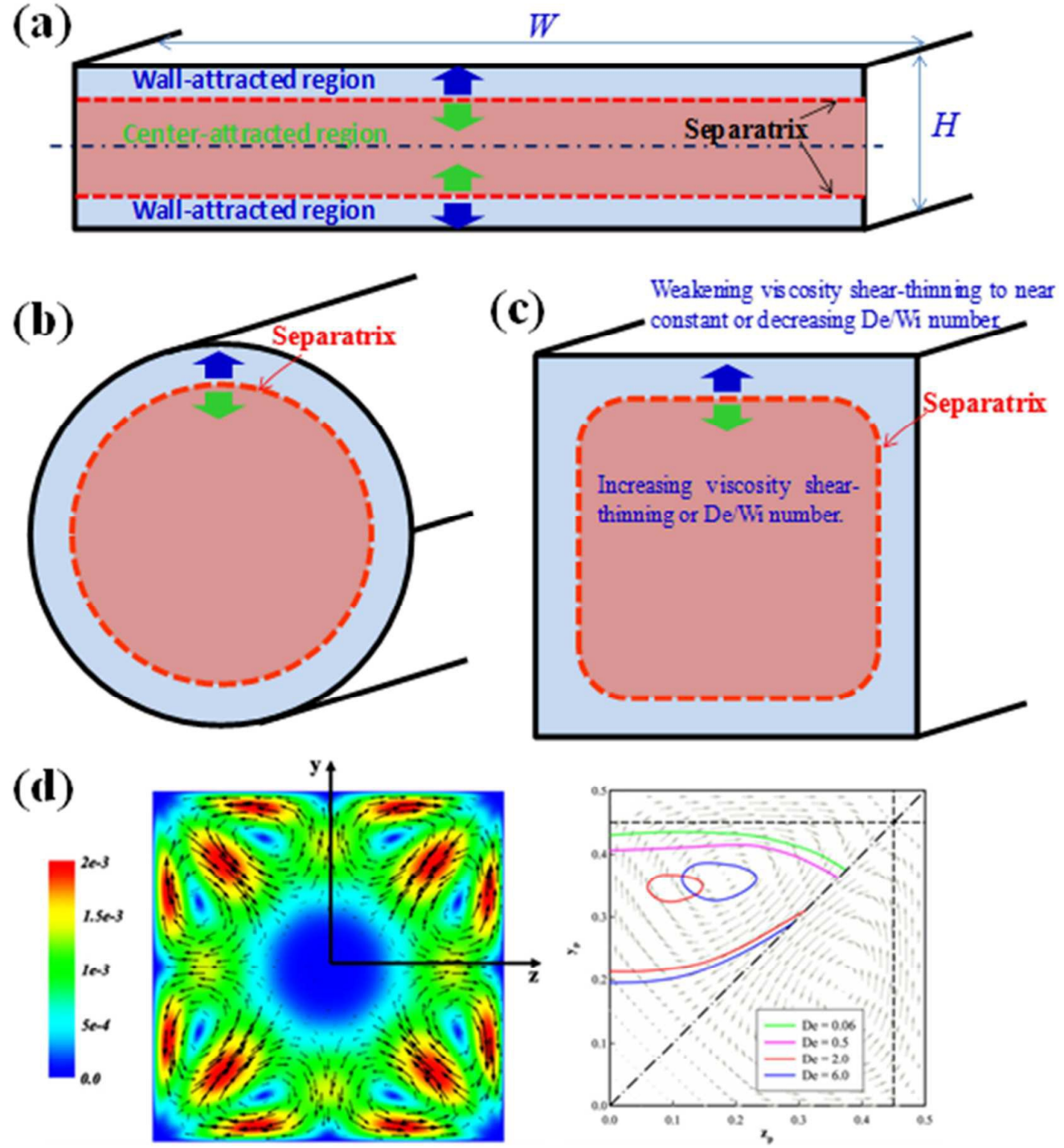

Figure 3 Separatrix in straight microchannels with (a) wide slit, W/H $>>1$; (b) circular cross section; (c) square cross section. Particles migrate towards different locations depending on their initial position. They migrate toward centerline when they are inside the separatrix, and migrate towards the wall/corner when they are outside 
of the separatrix. (d) Secondary flows induced by the second normal stress difference (left) and the unstable separatrices (right) ${ }^{55}$.

\subsection{Particle migration in elasto-inertial flow}

When particles migrate in viscoelastic fluid flow with a finite fluid inertia, the inertial effect becomes apparent. With a proper inertial effect, the particle focusing phenomenon becomes intriguing, and the throughput for particle focusing may be increased. The wall-lift force induced by the finite inertia can impair the corner attraction effect of elasticity, and equilibrium positions at corners become unstable, and particles focus only at the channel centerline, which is termed as elasto-inertial focusing ${ }^{56}$.

The concept of "elasto-inertial particle focusing" was first proposed to illustrate the 3D sheathless focusing in a straight square channel by Yang et al. ${ }^{47}$ They proposed three different focusing regions according to the importance of elasticity and inertia, as shown in Figure 4 (a). In inertia dominant flow, the particles are gathered at four equilibrium positions facing the center of each wall due to the inertial lift force. In elastic dominant flow, particles migrate to the lower first normal stress regions corresponding to the centerline and four corners under the pure elastic effects. The multiple equilibrium positions are not favorable in practical applications. Then, the equilibrium positions can be reduced to one at the center by increasing the flow rate and regulating the relative importance of inertial and elastic effects.

Meanwhile, the elasto-inertial particle migration with an increasing inertia was investigated in straight microchannels with circular ${ }^{57}$ and square ${ }^{58}$ cross sections, respectively, using a holographic technique. In a circular pipe flow in a PEO fluid with shearthinning property, particles can only be confined within the channel central area at lower flow rates, but disperse again as the flow rate increases ${ }^{57}$, as shown in Figure 4(b). Similarly, in a square microchannel in PEO fluid, under a balanced condition of inertia and elasticity, the particles can be focused tightly, but the increased flow rate and small blockage ratio are not in favor of particle focusing ${ }^{58}$, as shown in Figure $4(\mathrm{c})$. The re-disperse phenomenon is 
believed to be due to the increased inertial effects (the shear-gradient lift force directs to the channel wall) as well as the substantially decreased viscosity for shear-thinning fluid. However, in elasticity-dominant PVP fluid in a circular pipe flow ${ }^{57}$ or a square microchannel ${ }^{58}$, the particles migrate toward the tube center as flow rate, blockage ratio and channel length increase.

Besides the circular and square cross sections, elasto-inertial particle migrations in rectangular channels with different aspect ratios were studied. The continuous-flow sheathfree size-based particle separation in viscoelastic fluid in high width/height rectangular microchannels were realized ${ }^{59,60}$, Figure 4 (d). Multiple particle focusing lines were observed in rectangular channels with a high aspect ratio ${ }^{61} 62$, Figure $4(\mathrm{e}$ and $\mathrm{f})$. The reason is that as the inertial effect increases, the shear-gradient lift begins to take effect. As its direction points towards the channel wall, it may defocus the particles from the centerline.

Besides the widely used PEO and PVP viscoelastic aqueous solution, some other solutions are used as viscoelastic fluids. Since relatively high flow rates are needed to turn on inertial effects in elasto-inertial focusing, the throughput is higher than that in viscoelasticitydominant focusing. Moreover, if the solution is with a low viscosity but high elasticity, such as DNA or hyaluronic acid (HA) solutions, the throughput can be enhanced significantly.

Based on the larger size and longer relaxation time of DNA, particle focusing in DNA solution in good quality over a wide range of flow rates $(0.08-30 \mu 1 / \mathrm{min})$ was achieved ${ }^{63}$. Furthermore, Lim et al. ${ }^{64}$ reported successful "elasto-inertial" particle focusing at an extremely high flow rate $(50 \mathrm{ml} / \mathrm{min})$ in HA solution, which is the highest reported flow rate for particle viscoelastic focusing up till now. 
(a)

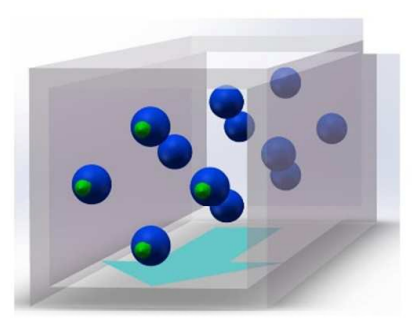

$\mathrm{Re}>\mathbf{0}, \mathrm{Wi} \approx 0$

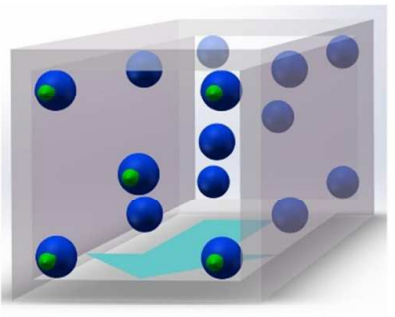

$\mathrm{Re} \approx \mathbf{0}, \mathrm{Wi}>\mathbf{0}$

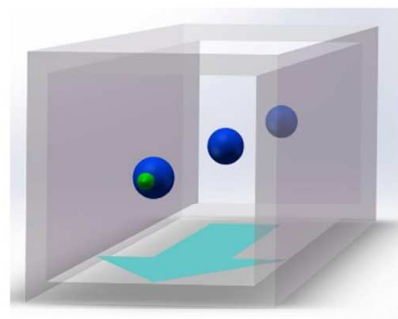

$\operatorname{Re}>\mathbf{0}, \mathbf{W i}>\mathbf{0}$

(b)
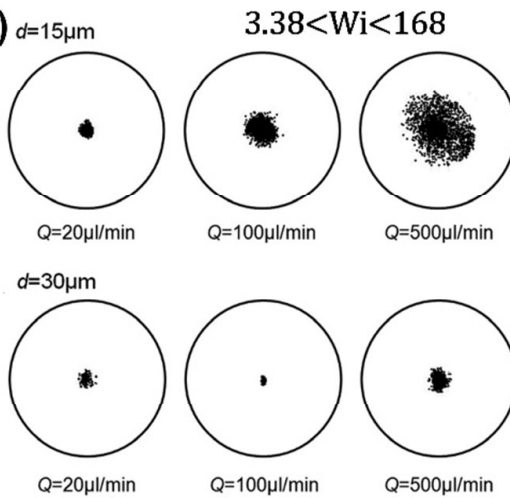

(d)
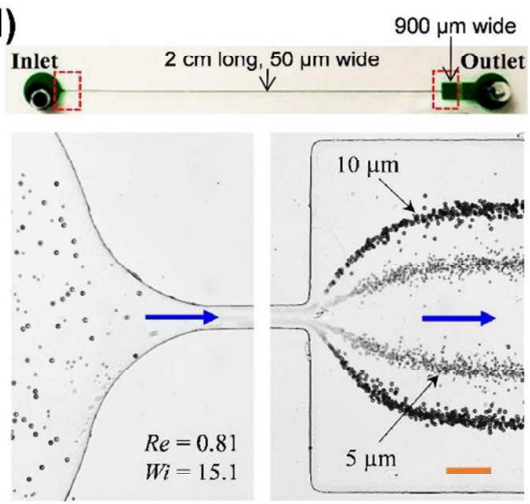

(f)

(c) ${ }_{\beta=0.1}$
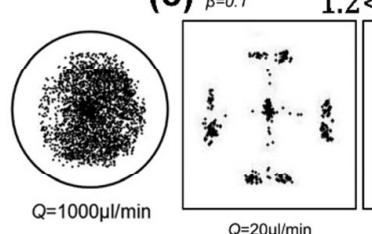

$2<\mathrm{Wi}<60$

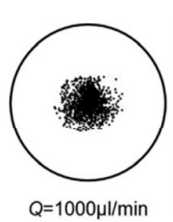

$\beta=0.17$
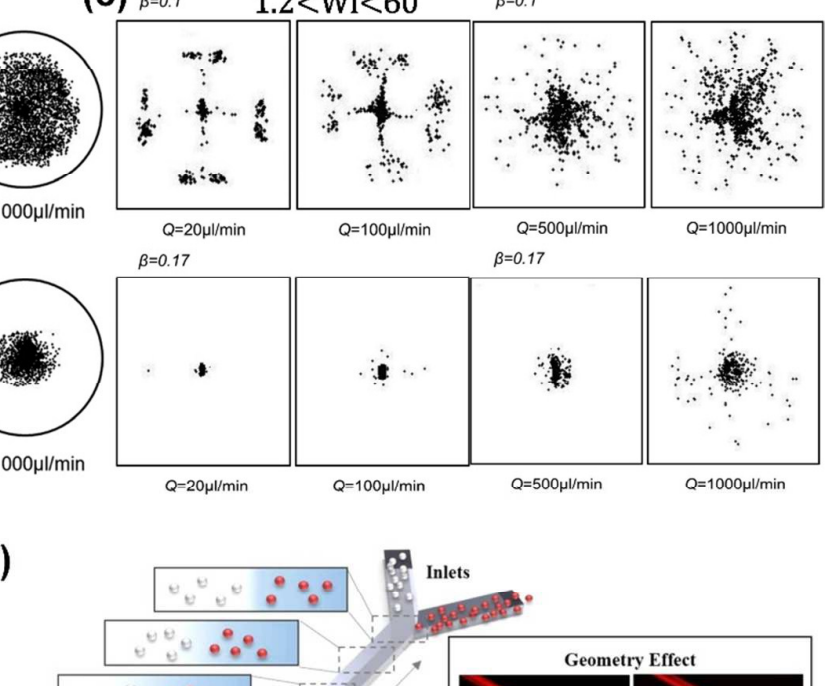

Inlets

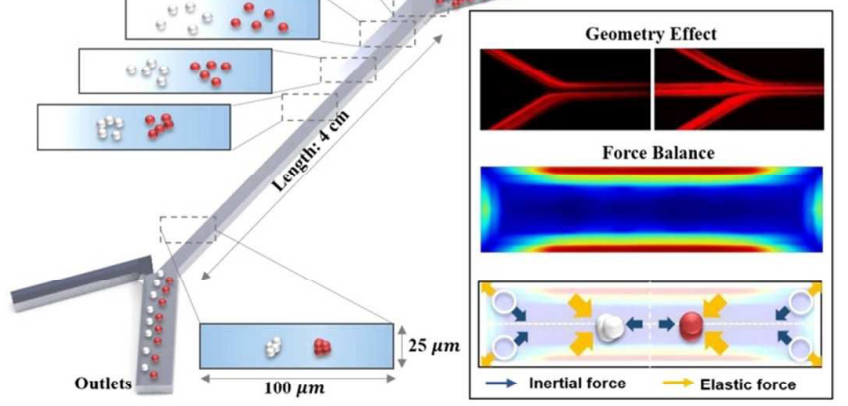

(e)

Reynolds Number $\left(\operatorname{Re}_{\mathrm{c}}\right)$

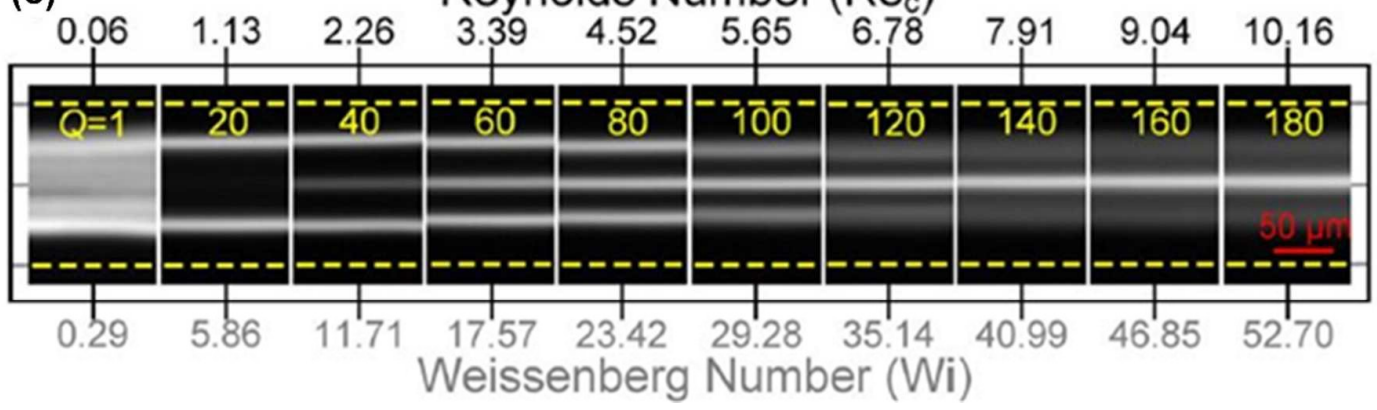

Figure 4 (a) Particle equilibrium positions in inertial dominant focusing, elastic dominant focusing, and elastoinertial particle focusing ${ }^{47}$. (b) Cross-sectional particle focusing in shear-thinning PEO fluid in a microscale pipe flow at different flow rates ${ }^{57}$. (c) Cross-sectional particle focusing in shear-thinning PEO fluid in a square 
microchannel at different flow rates ${ }^{58}$. (d) "Elasto-inertial" size-based particle separation in channel straight microchannel with a high width/height ratio in $1000 \mathrm{ppm}$ PEO fluid ${ }^{59}$. (e) Multi-train particle elasto-inertial focusing in straight rectangular channels with high aspect ratios at different flow rates ${ }^{61}$. (f) "Elasto-inertial" single-line to multiple-line particle focusing in a high aspect ratio channel ${ }^{62}$. 


\section{Applications}

The basic particle migrations in viscoelasticity-dominant flow and elasto-inertial flow in straight channels have been discussed above. The intrinsic viscoelasticity of fluid brings many intriguing and unique phenomenon on particle migration. In addition, viscoelasticityinduced particle migration in microfluidics can be effectively used in a wide range of applications, which will be elaborated in the following sections.

\subsection{Particle separation}

Separation of biological particles (DNA, cells, virus, bacteria, exosomes etc.) is highly important in various biomedical and biotechnological applications ${ }^{65,66}$, because they are promising biomarkers in the early detection and diagnosis of many diseases. Many researchers have developed the bioparticles separation techniques in viscoelastic fluid.

\subsubsection{Aid of sheath flow}

In viscoelastic fluid, particles are prone to migrate to the center of the channel under a proper elasto-inertial effect, and the migration speed is proportional to particle size. By pre-aligning all the particles with different sizes at the same initial position, the following size-dependent lateral migration speed will create a lateral gap between different sized particles. Therefore, particle separation based on lateral position difference can be achieved.

With the aid of sheath flow, elasto-inertial particle separation in PEO fluid in a square channel with high purity was realized ${ }^{67}$, as shown in Figure 5 (a). Viscoelastic sample suspension was injected from two side inlets, while the viscoelastic sheath flow was injected from the middle inlet, squeezing the particles to the sidewalls of the channel. The four inertial equilibrium positions are broken by the elastic forces, whose direction is away from the wall, leaving one equilibrium position in the centerline of the channel. As the inertial and elastic forces are both proportional to the particle sizes, larger particles migrated more quickly than smaller ones, resulting in their separation. Through this separation principle, platelets were 
separated from diluted whole blood with high purity (nearly 99.9\%). RBCs and WBCs with larger sizes migrated to the center of the channel, while platelets with smaller sizes remained focused along the side walls with a minimum lateral displacement.

(a)
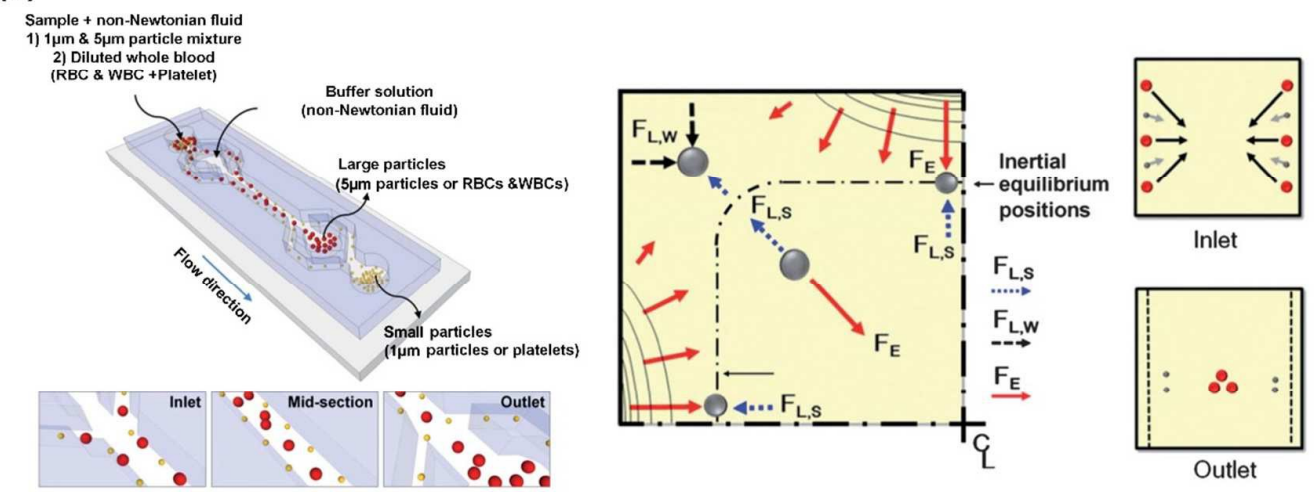

(b)

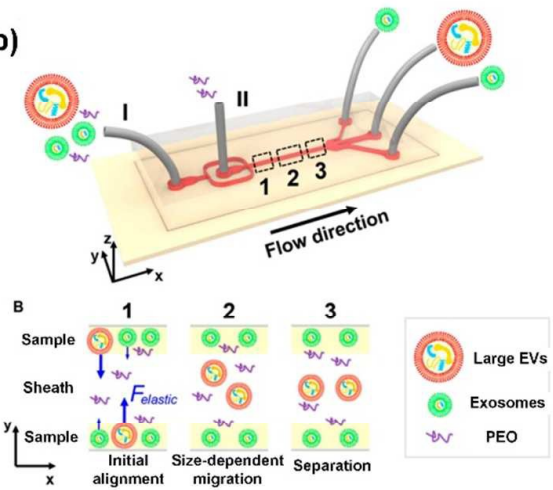

(c) Particle mixture

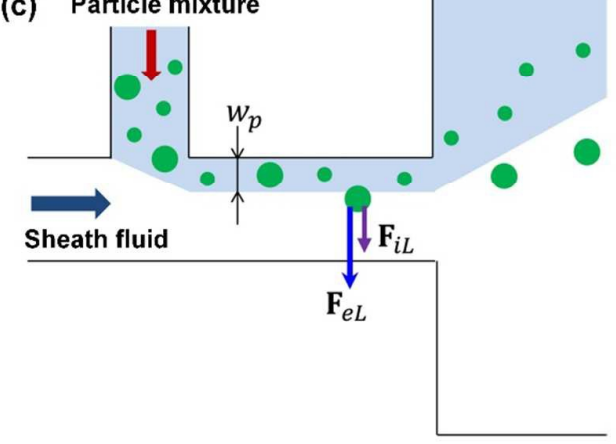

(d) Sheathless

Entrance region

Sample flow $\quad \therefore \quad \quad \because{ }^{\text {st }}$ stage: viscoelastic $3 \mathrm{D}$ focusing $\because$ (A) $\quad$ Bifurcation:initialization

(e)
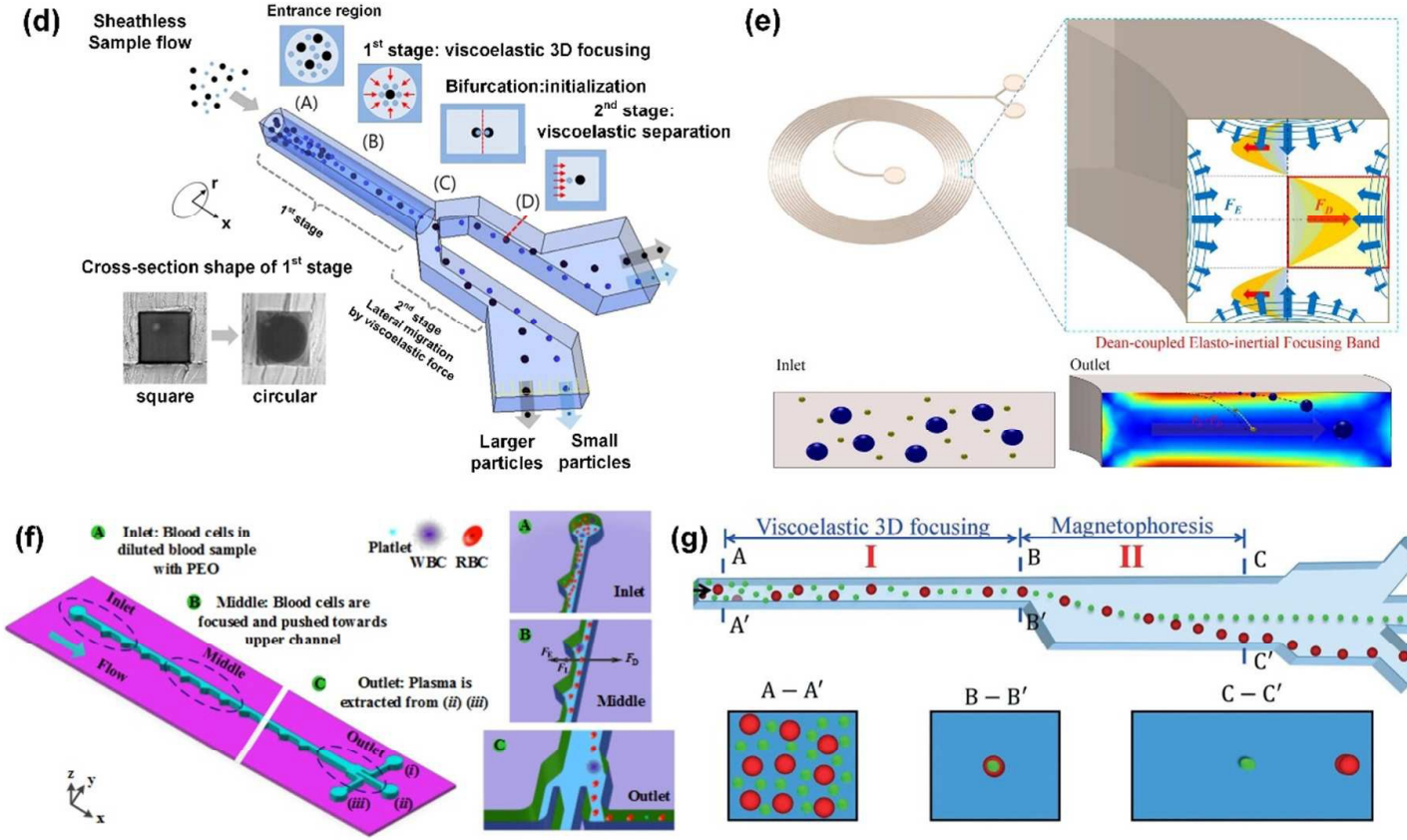

(g) $\underset{\mid \mathrm{A}}{\stackrel{\text { Viscoelastic 3D focusing }}{\text { I }}} \underset{\mid \mathrm{B}}{\stackrel{\text { Magnetophoresis }}{\text { II }}} \rightarrow$
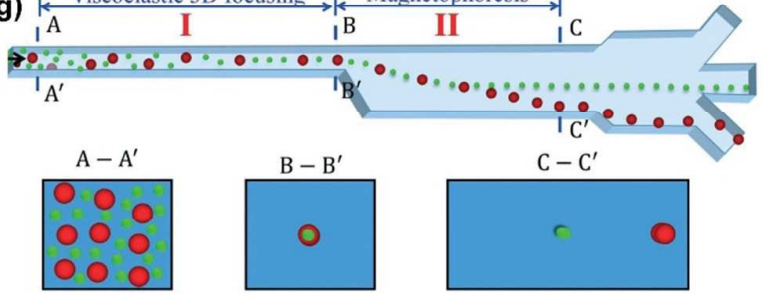
Figure 5. Particle separation in viscoelastic fluids. (a) Elasto-inertial particle separation in PEO fluid in a square channel with high purity ${ }^{67}$. (b) Elasto-inertial exosomes separation from cell culture media or serum in a continuous, size-dependent, and label-free manner in a straight channel ${ }^{68}$. (c) "Elasto-inertial pinched flow fractionation" (eiPFF) size-based particle separation in PEO solutions in a T-shaped microchannel ${ }^{69}$. (d) Sheathless particle focusing in a circular channel, followed by size-based particle separation using a symmetric bifurcation channel in elasticity dominant PVP fluid ${ }^{70}$. (e) Dean-flow-coupled elasto-inertial particle focusing and separation in spiral channel in PEO fluid ${ }^{71}$. (f) Sheathless, and high purity plasma extraction using deanflow-coupled elasto-inertial effect in ECCA channel ${ }^{72}$. (g) Viscoelastic nonmagnetic particle 3D focusing, followed by size-based negative magnetophoresis particle separation in viscoelastic ferrofluid ${ }^{73}$.

Based on the size difference of blood cells and bacteria, bacteria were separated successfully from whole blood using elasto-inertial effect in straight channel with the aid of sheath flow in viscoelastic fluid ${ }^{74}$. Similarly, Liu et al. ${ }^{68}$ successfully separated exosomes from cell culture media or serum in a continuous, size-dependent, and label-free manner with a high separation purity $(>90 \%)$ and a high recovery $(>80 \%)$ in a straight channel, as shown in Figure 5 (b). The proposed technique may serve as a versatile platform to facilitate exosome analyses in diverse biochemical applications.

Based on the same principle, Lu and Xuan ${ }^{69}$ proposed the "elasto-inertial pinched flow fractionation" (eiPFF) to separate particles by size in PEO solutions in a T-shaped microchannel, as shown in Figure 5 (c). They investigated in detail the factors influencing the separation performance in eiPFF method, including the PEO concentration, the flow rate and flow rate ratio of sample flow and sheath flow, the particle size, the aspect ratio of the channel. Furthermore, shape-based particle separation in the eiPFF was demonstrated ${ }^{75}$. Particles with spherical and peanut-shaped rigid particles of equal volume were separated in 1000ppm PEO solution, whereas separation cannot be realized in Newtonian fluid.

Apart from the normally used PEO solution, separation of particles with four different sizes in DNA solution was also achieved using the same mechanism ${ }^{63}$. Compared with the 
particle separation in Newtonian fluid, the separation resolution has been proven significantly enhanced in viscoelastic DNA fluid.

\subsubsection{Symmetric bifurcation channel}

Besides sheath flow, symmetric bifurcation channel were proposed to initially align particles along the sidewall to optimize the particle differential migration in viscoelastic fluid. Nam et al. ${ }^{70}$ realized particle focusing in a circular channel, followed by size-based particle separation using a symmetric bifurcation channel in elasticity dominant PVP fluid, as shown in Figure 5 (d). The difference in lateral displacements of particles was magnified by a sudden expansion region. Besides, they also realized the size-based elasto-inertial separation of RBCs, platelets, and $15 \mu \mathrm{m}$ polystyrene particles. Although the flow rate was limited, it was the first report to realize sheathless particle initialization and continuous label-free particle separation in viscoelastic flow. Moreover, they found that microchannel with a highaspect ratio in the first focusing stage and a low-viscosity polymer (HA solution) can improve the separation throughput ${ }^{76}$. Additionally, they successfully separated malaria parasite from WBCs with high recovery rate $(94 \%)$ and purity $(99 \%)^{76}$. They further modified the device using a commercially available circular capillary tube for particle pre-focusing ${ }^{77}$, and realized separation of MCF-7 cells from leukocytes with a high recovery rate and a high purity based on the same principle.

\subsubsection{Aid of Dean flow}

In a curved or a straight channel with contraction and expansion cavity arrays, the Dean flow appears and can be adjusted to enhance particle separation.

In a spiral channel, when the inertia effect is not negligible, particles are affected by the "dean-flow-coupled elasto-inertial" effect. Particles can be focused in a 3D manner or 
separated by the combined effects of three forces: inertial lift force $F_{\mathrm{L}}$, including the sheargradient lift force $\left(F_{\mathrm{LS}}\right)$, wall-repulsion force $\left(F_{\mathrm{LW}}\right)$, the Dean drag force $F_{\mathrm{D}}$ resulting from the curved channel geometry, and elastic force $F_{\mathrm{E}}$ induced by the nature of the viscoelastic medium. Based on the effect, different sized particles are focused differentially at the outlet, and particle separation was realized ${ }^{71}$, as shown in Figure 5 (e). The differential migration and separation of particles was also achieved in a novel integrated 2-spiral microdevice in viscoelastic fluid ${ }^{78}$. The throughput and separation efficiency have been improved significantly.

In addition to the curved structure, the straight channel with expansion-contraction cavity arrays (ECCA channel) can also induce a Dean-like flow. Based on the dean-flowcoupled elasto-inertial effects, we demonstrated 3D sheathless particle focusing in ECCA channel ${ }^{79}$. Interestingly, in the viscoelastic fluid, particles are 3D focused on the opposite cavity side, opposed to that in a Newtonian fluid. Furthermore, using the same channel design, we achieved sheathless, and high purity plasma extraction, as shown in Figure 5 (f) ${ }^{72}$. In the experiments, all the blood cells (including WBCs, RBCs, and platelets) were focused to a single line and filtrated out of blood stream. After two series of processing, the plasma purity can reach as high as $99.99 \%$. Furthermore, by integrating the ECCA channel section and a straight channel section, our group has also realized sheathless Dean-flow-coupled elastoinertial particle focusing at the first stage and size-based differential particle separation at the second stage ${ }^{80}$.

Precise manipulation of sub-micrometer to nanometer-scale particles in passive microfluidics is technically challenging because the Brownian motion may become dominant once the particles size is reduced to the nanometer-scale. However, recently it has been proven that it may be achievable by employing fluid viscoelasticity ${ }^{81,82}$. The submicron polystyrene (PS) beads were successfully focused in the center and corners in a rectangular 
channel in PEO solution by the pure elastic effect, and flexible DNA molecules were also focused in the centerline of the channel by the integration of elastic force and wall lift force originating from the flexibility of DNA molecules ${ }^{81}$. Flow focusing of sub-micrometer particles in cylindrical channels has been reported by De Santo et al. ${ }^{82}$. In addition, they proposed a simple theoretical model that supports the experimental evidence, and links the trapping force to a dimensionless parameter comparing viscoelastic normal forces and Brownian forces. Using a double spiral channel, $\lambda$-DNA molecules/blood platelets were focused and separated successfully in a low molecular weight PEO solution ${ }^{83}$. The superior manipulation capacity by viscoelastic fluid on submicro to nano particles is a significant advantage of viscoelastic microfluidics compared with other passive microfluidic manipulation technologies.

\subsubsection{Aid of magnetophoresis}

Viscoelastic particle migration is one of the passive methods, when integrated with other active methods, the device can be more versatile, and provide more precise control of target bio-particles. By integrating positive magnetophoresis with viscoelastic particle focusing, for the first time, Del Giudice and his coworkers ${ }^{84}$ realized the high efficiency magnetic particle separation in a H-shaped channel in PAM solution. The magnetic particles were firstly prefocused at the centerline of rectangular channel by viscoelastic force, and then attracted laterally to the sheath fluid by a permanent magnet. The pre-focusing in viscoelastic fluid prevents particles from getting stuck on the wall, and flowing out to the undesired outlet in the magnetic deflection section. Using this method, the magnetic and non-magnetic particles are separated with a high efficiency. Later, Zhang et al. ${ }^{73}$ and Min et al. ${ }^{85}$ proposed similar methods combining viscoelastic focusing and negative magnetophoresis to separate nonmagnetic particles in the ferrofluid with viscoelastic base medium. The viscoelastic force was 
used to align particles at the channel centreline before the magnetic region, and sizedependent magnetophoretic force was applied on particles to separate particles by size, as shown in Figure $5(\mathrm{~g})$. The integration of viscoelastic particle migration and active methods makes the viscoelastic microfluidics more flexible and versatile, with potential more functionality.

\subsection{Cell deformability measurement and alignment}

(a)
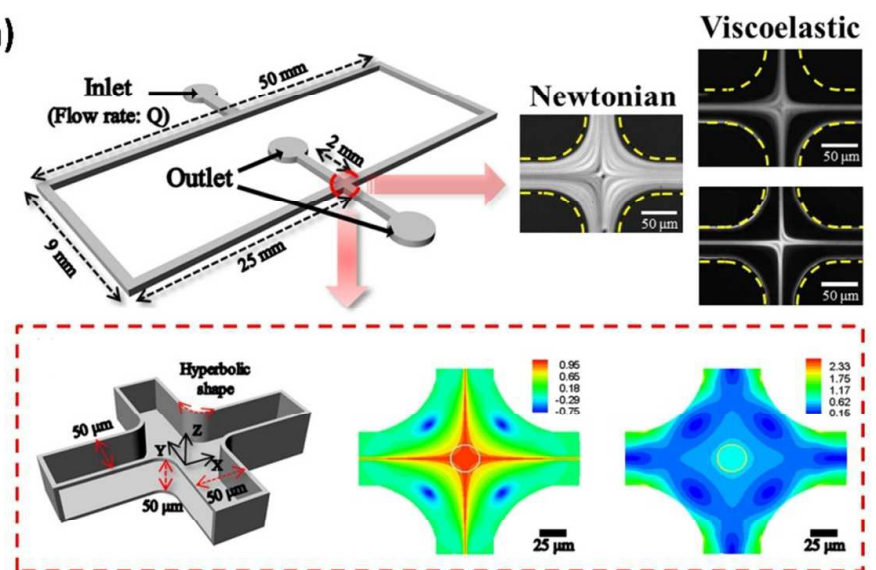

(c)
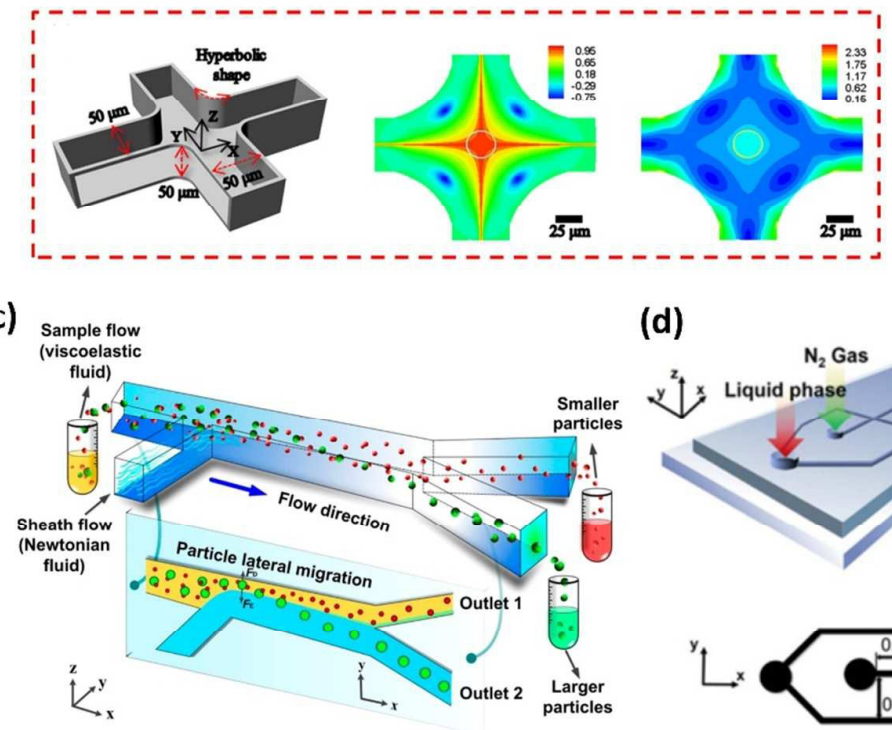

(d)
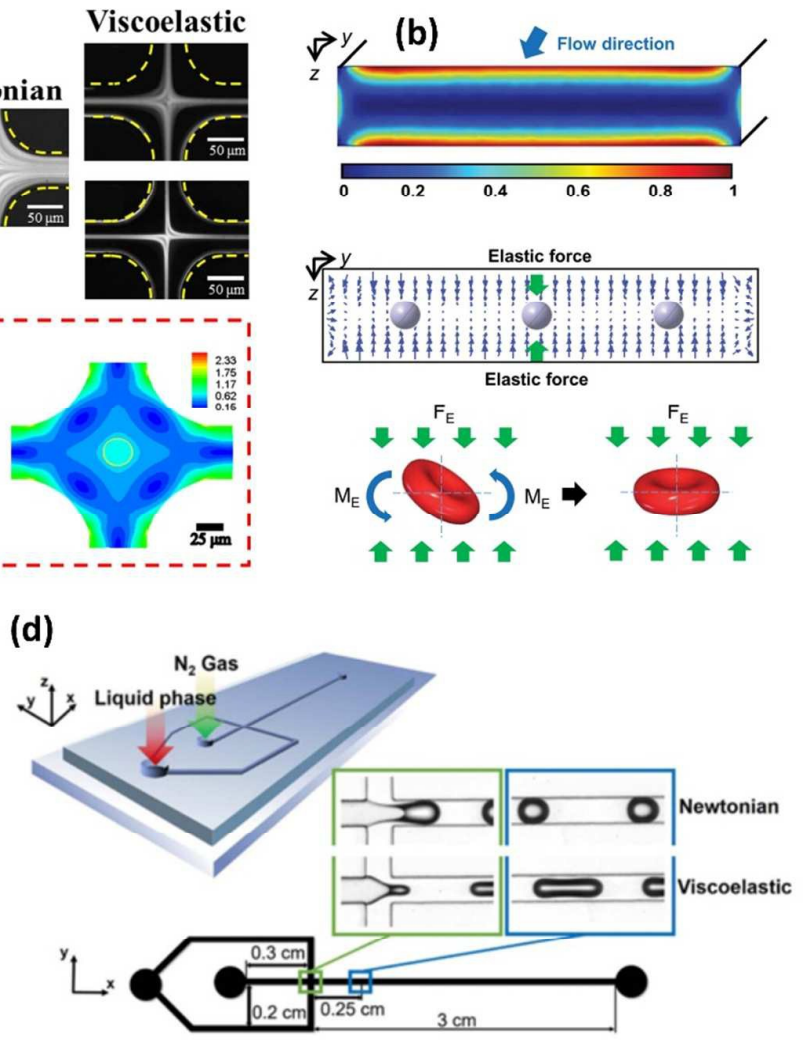

Figure 6. Applications of viscoelasticity-induced particle migration in microfluidics. (a) Viscoelastic 3D particle pre-focusing, followed by cell deformability measurement based on the extensional field in rectangular channel in PVP solution ${ }^{86}$. (b) Sheath-free RBC vertical concentration and positioning induced by fluid viscoelasticity in a rectangular channel ${ }^{87}$. (c) On-chip particle washing using coflow of a PEO-contained viscoelastic fluid and a Newtonian fluid ${ }^{88}$. (d) Elastic effects on bubble generation processes in a microchannel 89. 
Cell deformability is a promising label-free biomarker for the diagnosis of diseases ${ }^{90,91}$. The alteration in the deformability of cells compared with healthy cells can be utilized to detect blood diseases ${ }^{92}$, cancers $90,91,93,94$ and so on. For example, the alteration in the deformability in RBCs can help diagnose the malaria, sickle cell anemia, and diabetes diseases. The reduction in stiffness of the cells in abnormal tissue is an indication of cancer. Compared with the conventional molecular-based biomarkers, which require pre-processing steps, costly antibodies or dyes, the deformability as a biomarker is label-free, simpler, cheaper and has the potential for automated measurement ${ }^{95,96}$.

Villone et al. has studied the lateral migration of deformable particle in tube flow of Newtonian and viscoelastic media through 3D finite element method numerical simulations ${ }^{97}$. They found that if the particle is initially not at the channel axis, it attains an asymmetric shape. In a Newtonian liquid, the migration is always directed towards the tube axis. In a viscoelastic liquid, the migration direction and velocity depend on the competition among particle deformability, fluid elasticity, or fluid viscosity shear thinning. In a certain range of parameters, an unstable radial position appears, which separates the region where the migration is directed towards the axis from the region where it is directed towards the wall.

The migration phenomenon in rectangular channels is different. As we know that the rigid spherical particles in viscoelastic fluid without inertia in rectangular channels migrate towards the centerline and corners, while the deformability effect results in additional wall repulsion forces to exert on deformable particles or cells and selectively entrains them at the centerline. Based on this principle, Yang et al. ${ }^{98}$ successfully extracted WBCs (collected along the channel corners) from RBCs (concentrated at the centerline of the channel) with a purity of $35 \%$ from a dilute input solution with $\mathrm{WBC} / \mathrm{RBC}$ ratio of $0.17 \%$.

Recently, based on the viscoelastic 3D particle pre-focusing, cell deformability measurement with a high detection efficiency using the extensional flow field in rectangular 
channel in PVP solution was realized ${ }^{86}$, as shown in Figure 6 (a). After initially homogenization of cell trajectories, the RBC will be delivered to a stagnation point for stretch measurement. The detection efficiency was improved compared with that in Newtonian fluid. They also found that after heat treatment, RBC deformability can be changed; after nutrient starvation in human mesenchymal stem cells, their deformability will be decreased. Using the similar method, Kim et al. performed shape measurement of ellipsoidal particles in the crossslot microchannel utilizing viscoelastic particle focusing ${ }^{99}$. The shapes of ellipsoidal particles with various aspect ratios were successfully measured. This method can break the limitation of particle aggregation or uncertainty out-of-plane arrangement of particles in conventional methods such as optical microscopy, and can be useful in a wide range of applications such as shape measurement of nonspherical cells.

RBCs are normally arbitrarily oriented in Newtonian fluid, which is not favorable for accurate cell monitoring and counting in optical imaging system. Sheath-free RBC vertical concentration and positioning induced by fluid viscoelasticity in a rectangular channel has been demonstrated ${ }^{87}$, as shown in Figure 6 (b). Monitored by a digital holographic microscopy, RBCs can be in high uniformity under the effect of the elastic force, and the cell overlapping can be reduced. Therefore, the out-of-focus blurring can be eliminated, and the detection sensitivity can be increased.

Moreover, the abnormal erythrocyte deformability which is potential for label-free diagnosis of hematological diseases was studied ${ }^{100}$. By employing digital in-line holographic microscopy (DIHM), they investigated the lateral migration of human erythrocytes induced by viscoelastic fluid flow in a rectangular microchannel. Different from rigid spheres and hardened erythrocytes, deformable normal erythrocytes become more dispersed in the channel center area as the flow rate increases. Additionally, normal erythrocytes have a higher angle of inclination than hardened erythrocytes in the region near the side-walls of the 
channel.

Most recently, a striking collective swimming of bovine sperm in dynamic clusters, enabled by the viscoelasticity of the fluid was reported ${ }^{101}$. Sperm oriented in the same direction within each cluster, and cluster size and cell-cell alignment strength increased with viscoelasticity of the fluid. The collective swimming induced by elasticity may facilitate sperm migration and contribute to successful fertilization. This work implies that the fluid elasticity may be of great importance in biological function, since almost all biological fluids are viscoelastic in nature.

For the first time, Holzner et al. investigated the elasto-inertial focusing of mammalian cells and bacteria using low molecular and low viscosity PEO solutions ${ }^{102}$. This kind of PEO solution exhibits negligible shear thinning, and particles can be focused over a wide range of elasticity numbers and a large range of Reynolds numbers. In addition, the influence of blockage ratio, volumetric flow rate, cell concentration, and polymer chain length was assessed. The current method can be used to focus cells without inducing bodily rotation, which is especially useful in morphology-based analysis of disease-infected cells and suggests the realization of an optofluidic platform for imaging and cytometric analysis in the short term.

\subsection{Particle Solution exchange}

Particle focusing and separation using sample-sheath flow with the same fluid property (either Newtonian fluids or non-Newtonian fluids) has been explored extensively, while sample-sheath flow with different fluid properties has rarely been studied. Jayaprakash et al. 103 investigated the dynamics of aqueous droplets of different size and viscosity at the interface of a coflowing stream of immiscible oils (silicone oil as primary and mineral oil as secondary continuous phases). They elaborated that the competing noninertial lift and interfacial tension forces governs the interfacial migration of the droplets. Sorting of droplets 
based on size contrast is demonstrated as well. Later, they reported the dynamical migration behavior of rigid polystyrene microparticles at an interface of coflowing streams of primary (aqueous) and secondary (oils) immiscible phases at low Reynolds numbers in a microchannel ${ }^{104}$. They found that the migration criterion depends on the sign of the spreading parameter and the presence of surfactant at the interface, and the interfacial perturbation can cause detachment of microparticles from the interface. Moreover, size based sorting of microparticles was demonstrated. Recently, Yuan et al. ${ }^{105}$ and Ha et al. ${ }^{106}$ have studied the lateral migration behaviors of particles using the co-flow configuration of viscoelastic and Newtonian fluids. Ha et al. ${ }^{106}$ implemented particle separation using $\lambda-D N A$ viscoelastic and Newtonian fluids. Instead of using $\lambda-D N A$ to form viscoelastic fluid, Yuan et al. ${ }^{105}$ have investigated particle lateral transfer from poly(ethylene oxide) (PEO)-containing viscoelastic fluid to a Newtonian fluid. Furthermore, the critical particle size for efficient particle washing, and on-chip washing of Jurkat cells using the co-flow of viscoelastic fluid and Newtonian fluid was presented ${ }^{88}$, as shown in Figure 6 (c). This technique may be a safer, simpler, cheaper, and more efficient alternative to the conventional centrifugation methods and may be applied for a wide range of biomedical applications. Recently, the viscoelastic/Newtonian interfacial effect has been investigated, and a size-based separation of microparticles in co-flow of Newtonian (water or PBS) and viscoelastic fluids (PEO) were illustrated with the aid of interfacial effect ${ }^{107}$. Small particles cannot traverse the interface between Newtonian and viscoelastic media due to the dominance of wall-directed interfacial elastic lift forces, whereas large particles cross the interface due to the dominated inertial lift forces. Additionally, they realized the separation of Staphylococcus aureus $(1 \mu \mathrm{m})$ from platelets $(2-3 \mu \mathrm{m})$ with high efficiency and purity.

\subsection{Rheometry-on-a-chip}

Microfluidic viscometers for the shear rheology and extensional rheology of complex fluids 
and biofluids have been investigated extensively recently ${ }^{108-110}$. These miniaturized devices are helpful especially in the case of low viscosity, weakly elastic fluids, low sample volume and so on. Rheometry-on-a-chip has been proposed by exploiting the characteristics of viscoelastic fluid in microfluidic channels. By testing creep recovery in a microfluidic device, the measurement of relaxation time of viscoelastic fluids at low strain has been realized ${ }^{111}$. Microfluidic rheometric devices that are capable of measuring fluid relaxation times down to $1 \mathrm{~ms}$ have also been proposed by Zilz et al. ${ }^{112}$ based on the scaling behaviour of the onset of a purely-elastic flow instability in a microfluidic serpentine channel. This microfluidic rheometric device can assess lower molecular weight materials, solvent viscosities or concentrations than that using the state-of-the-art commercial rheometers. Besides, Del Giudice et al. ${ }^{113}$ found an effective way to measure relaxation times of viscoelastic fluids based on the particle migration phenomenon occurring when the suspending viscoelastic fluid flows in straight microfluidic channels, which does not need a calibration curve, and more easily detectable compared with conventional techniques. Two glycerol-water solutions on PEO and PAM solutions at various concentrations were tested. Later, they used a homemade $\mu$-rheometer based on the viscoelasticity-induced cross-flow migration to measure a characteristic relaxation time of polyelectrolytes solutions ${ }^{114}$. Relaxation time as small as 60 $\mu$ s was detected, which is the highest resolution compared with the conventional and other $\mu$ rheometrical techniques. Results from two different microrheometrical techniques: relaxation times in shear flow measured through the $\mu$-rheometer based on the viscoelastic alignment of particles in a straight microchannel, and in extensional flow measured in a microfluidic optimized cross-slot configuration based on the onset of the flow-induced birefringence were compared ${ }^{115}$. It is found that microfluidic techniques can capture very small relaxation times of dilute polymer solutions, and there is a good agreement from the two platforms. The advances in microfluidic rheology will help us better understand these complex fluids, and 
uncover their properties, as well as mechanisms in relation to the behaviors of living biofluids.

\subsection{Other applications (Mixing, heat transfer, droplet generation and sorting)}

Efficient mixing is required in a wide range of microfluidic applications, such as chemical synthesis and micro or nanoparticle production. Microfluidic flows are typically laminar because of the low Reynolds number $R_{\mathrm{c}}$. The viscoelasticity of polymer solutions can generate unstable flows even when $R_{\mathrm{c}}$ is very small and can significantly enhance mixing efficiency. Based on the chaotic vortex dynamics of a viscoelastic flow, an efficient microfluidic mixer was proposed by Hong et al. ${ }^{116}$. In viscoelastic fluid, as the Reynolds number $R_{\mathrm{c}}$ and Weissenberg number $\mathrm{W}_{\mathrm{i}}$ both increase to a certain value, the instability of elasto-inertial flow will appear, leading to chaotic vortices in the side wells. This kind of instability can enhance the mixing of adjacent fluid streams. In order to improve mixing, a channel that had sudden expansion and contraction along with teeth patterns along the channel walls was employed by Julius et al. to perform viscoelastic mixing ${ }^{117}$. They also completed on-chip erythrocytes lysis based on the efficient mixing effect. The erythrocytes are gradually lysed along the channel, leaving WBCs at the outlet.

By employing the instability of a viscoelastic fluid with a lower viscosity, fluid mixing at subcritical Reynolds numbers was induced, thus the heat transfer in a serpentine channel was improved ${ }^{118}$. Due to the low pumping cost of the fluid, it would be an attractive cooling method, which can be used in industrial applications such as next generation photonic devices for enhanced local hot-spot cooling. Heat transfer in both Newtonian and nonNewtonian viscoelastic fluids was investigated by embedding Ti-Pt films on the bottom of channel wall as temperature sensors ${ }^{119}$. They concluded that due to the instability of the viscoelastic fluid, which can induce fluid irregular motion, it has a better heat transfer performance compared with that of Newtonian fluid.

Viscoelastic fluid has also been used for droplet generation and sorting. Droplet 
separation was achieved based on the intrinsic viscoelastic fluid properties ${ }^{120}$. Droplets with different intrinsic viscous and viscoelastic properties relative to the continuous oil phase would migrate to high or low shear rate regions. This passive droplet sorting method is simpler and in lower cost, and can be potentially used in the chemical or biological fields where droplets with different intrinsic viscoelastic properties need to be separated. The effect of elasticity on bubble generation processes in a microchannel was also investigated ${ }^{89}$, as shown in Figure $6(d)$. They found that even a small amount of polymer can have significant effect on bubble generation. Due to the instability of the flow, the generated bubble sizes are fluctuated. Compared with the bubble generated in Newtonian fluid, the bubble were thinner along the minor axis in the viscoelastic fluid due to the viscoelastic effect. 


\section{Conclusion and Perspectives}

In this review, we discussed particle migration in viscoelastic fluids mainly from the aspect of applications. The hydrodynamic forces and the basic particle migration in viscoelasticitydominant and elasto-inertial flow in straight channels were elaborated. After that, the applications of viscoelasticity-induced particle migration in microfluidics, including particle separation, cell deformability measurement and alignment, particle solution exchange, Rheometry-on-a-chip and many other applications in microfluidics were comprehensively reviewed. In this section, we propose several potential future research directions that probably deserve more attention and efforts from the microfluidic community.

\subsection{More fundamental studies in viscoelastic fluid are needed.}

Due to the complexity of the problem, the particle behaviors in viscoelastic fluids are strongly influenced by channel cross sectional shape, elasticity effects, inertial effects, shearthinning viscosity, secondary flows, and blockage ratio etc. To uncover the principle of particle behaviors in viscoelastic fluid, both numerical and experimental studies have been extensively conducted. However, there are still many discrepancies between numerical results with experiments, or experimental results from different literatures. For example, Del Giudice et al. ${ }^{53}$ observed that particles were focused at the centerline of square microchannel in a aqueous solution of PVP (polyvinylpyrrolidone, $8 \% \mathrm{wt}$ ) when Deborah number (De) is 0.2 . In contrast, for particles suspending in the PVP solution with similar rheological properties, Yang et al. found that particles migrates towards the centerline as well as four corners under the same Deborah number ${ }^{47}$. This indicates that, the exact relationship between fluid rheology and particle migration is still unclear. The hydrodynamic forces on particles, particle trajectories, the correlation of fluid rheology and particle migration, or particle behaviors in coflow of fluids with the same or different rheological properties needs further quantitative investigation. 
Migration of sub-micro or nano-sized biological particles (DNA, protein, virus, bacteria, exosomes, etc.) is highly important in various biomedical and biotechnological applications, because these bioparticles are promising biomarkers in the early detection and diagnosis of many diseases. However, the migration of nano-scale particles in microfluidics is rarely investigated from both theoretical and experimental aspects because the Brownian motion effect may be significant once the particles size is reduced to nano-scale. Recently, researchers have demonstrated that viscoelastic fluid has the potential to realize sub-micro or nanoparticle migration, which may open a new gate through the territory of nano-scale particles (DNA, protein and virus etc.).

\subsection{Particle interaction and the associated particle dynamics in viscoelastic fluid need} further investigation.

Particle interaction in inertial manipulation has been extensively studied. The dynamics of the particle-particle interactions was studied, and a mechanism for the dynamic self assembly process was revealed ${ }^{121}$. It is found that inertial lift forces and a parabolic flow profile stabilize interparticle spacings that otherwise would diverge to infinity due to viscous disturbance flows. With increasing concentration of the suspension the spacing is influenced by particle crowding effects until stable trains are no longer observed ${ }^{122}$. Moreover, Humphry et al. presented that both the location and the number of focusing positions depend on the number of particles per unit length along the channel ${ }^{123}$. This axial number density is a function of both the channel cross-section and the particle volume fraction. Reece et al. have explored equilibrium focusing behavior as a function of channel geometry and particle concentration ${ }^{124}$. It is concluded that particle buckling scales weakly with concentration and strongly with channel geometry.

However, very few works have been reported for particle interactions in viscoelastic fluid. We previously investigated the effects of blood hematocrit on the viscoelastic focusing 
behaviour in ECCA channel, and concluded that the higher the blood hematocrit, the more significant the interaction between blood cells, and the worse the cell focusing quality ${ }^{72}$. Holzner et al. ${ }^{102}$ also found that as cellular concentration increases, the cell radial distributions are broader, and the cell focusing efficiency is decreased. They explained that for more concentrated cell suspensions, the inter-cell space on the centerline of the channel decreases, and they compete for the same space around the channel centreline. Besides, the variation in the dimension of cell population generates eventually random cell clumps, which destroy any order. It's also found that the high volume fraction decreases the shear rate, weakening the effects of both shear thinning and elastic normal stresses, and the annular 'particle-free zone' becomes smaller as well ${ }^{36,125}$.

Since the particle-particle interaction directly impairs the viscoelastic focusing and separation performance. Determining the suitable cell concentration to maximize the throughput of microfluidic device, as well as maintaining the proper functionality is still challenging. The theoretical models to evaluate the effects of particle-particle interactions on particle viscoelastic focusing and extensive experimental quantification are still needed.

\subsection{Viscoelastic particle migration based on particle shape or deformability}

Shape is an important indicator for bioparticle separation as well, and it varies with cell type, cycle, and state, etc. It provides useful information in bioparticle identification, cell synchronization, disease diagnostics et al. Cell deformability is a promising label-free biomarker for the diagnosis of disease. The alteration in the deformability of cells compared with healthy cells may be utilized to diagnose blood disease, cancers and so on. Viscoelastic particle migration based on particle shape or deformability including the theoretical or numerical predictions, and experimental studies should be a promising research direction in the near future.

\subsection{Realize real "label-free" bio-particle migration}


The viscoelasticity-induced particle migration itself can be considered "label-free", compared with the conventional molecular-based biomarkers, the viscoelasticity-induced particle migration does not require costly antibodies or dyes. The particle size, shape, or deformability is the intrinsic label-free biomarker, which is simpler and cheaper. However, as the viscoelastic fluid is formed by dissolving polymers in Newtonian fluid. The polymer molecules always contact with particles in the medium. Polymer has been entitled as biomaterial for a long time ${ }^{126}$, and has been used for drug compounding ${ }^{127,128}$ and a wide variety of cosmetic and personal care products. In addition, it has also been used as a biocompatible modifier for a variety of enzymes and proteins to maintain their intrinsic activities ${ }^{129}$ or to improve its blood-compatible properties ${ }^{130}$. Recently, aqueous solutions of polymer have been widely used in microfluidics and for a wide range of bio-medical experiments elaborated above ${ }^{60,67,87}$. Although it is noteworthy that aqueous solutions of polymer are hospitable to living cells, it is not sure that any kind of polymer or polymer with any molecular weight is biocompatible and nontoxicity ${ }^{131}$. The influence of viscoelastic fluid on cells viability in microfluidics was rarely studied. Only in our previous experiments, the cells viability before and after solution exchange experiments were tested using viability assay method ${ }^{88}$. The cells' viability before experiment is about $96.9 \%$. The viability of cells from inlet after $2 \mathrm{~h}$ is about $96.5 \%$, while the viability of cells from outlet after PEO exposure is about $91 \%$. The results show that PEO has little effect on the cells' viability in short term. Its influence on viability and phenotype of cells on long term and its interfere with reagents is still unclear, which deserves more attention from scientific community. However, the elimination of PEO from blood plasma, cell suspension or aqueous solution is another technical barrier for the wide application of viscoelastic fluid on cell manipulation and separation. 
To minimize the effects of polymer moleculars on bioparticles, the target particles should be isolated from the viscoelastic fluids immediately after the operation. A solution exchange technique which uses the coflow of viscoelastic fluid and Newtonian fluid may be one of the promising in-line methods to wash target particles. However, it is still facing the limitation of throughput and washing efficiency. Therefore, more novel microfluidic technologies to change the solution and wash bioparticles in a high throughput are still in demand.

Besides, various body fluids such as blood, saliva, DNA solutions, cytoplasm, et al., exhibit viscoelastic properties. By utilising the natural viscoelasticity of these body fluids, the polymer label problem may be avoided. Thus, investigation of particle/cell migration in the natural body fluids and tailoring the particle viscoelastic migration principle for biomedical applications may deserve attention. 


\section{Acknowledgements}

This work is supported by the National Natural Science Foundation of China (Grant No.51705257), the Natural Science Foundation of Jiangsu Province (BK20170839), the Australian Research Council (ARC) Discovery Project (Grant No. DP180100055)) and the University of Wollongong-China Scholarship Council joint scholarships. 


\section{References}

1. S. Nagrath, L. V. Sequist, S. Maheswaran, D. W. Bell, D. Irimia, L. Ulkus, M. R. Smith, E. L. Kwak, S. Digumarthy and A. Muzikansky, Nat., 2007, 450, 1235.

2. A. A. S. Bhagat, H. Bow, H. W. Hou, S. J. Tan, J. Han and C. T. Lim, Med Biol Eng Comput, 2010, 48, 999-1014.

3. N. Pamme, Lab Chip, 2007, 7, 1644-1659.

4. P. Sajeesh and A. K. Sen, Microfluid Nanofluid, 2014, 17, 1-52.

5. D. R. Gossett, W. M. Weaver, A. J. Mach, S. C. Hur, H. T. K. Tse, W. Lee, H. Amini and D. Di Carlo, Anal Bioanal Chem, 2010, 397, 3249-3267.

6. D. Di Carlo, Lab Chip, 2009, 9, 3038-3046.

7. X. Xuan, J. Zhu and C. Church, Microfluid Nanofluid, 2010, 9, 1-16.

8. G. M. Whitesides, Nat., 2006, 442, 368-373.

9. J. Nilsson, M. Evander, B. Hammarström and T. Laurell, Analytica chimica acta, 2009, 649, 141-157.

10. L. J. Guo, X. Cheng and C.-F. Chou, Nano letters, 2004, 4, 69-73.

11. J. J. Benítez, J. Topolancik, H. C. Tian, C. B. Wallin, D. R. Latulippe, K. Szeto, P. J. Murphy, B. R. Cipriany, S. L. Levy and P. D. Soloway, Lab Chip, 2012, 12, 4848-4854.

12. Y. Kang, D. Li, S. A. Kalams and J. E. Eid, Biomed. Microdevices, 2008, 10, 243-249.

13. M. Li, S. Li, W. Cao, W. Li, W. Wen and G. Alici, J Micromech Microeng, 2012, 22, 095001.

14. S. Yan, J. Zhang, G. Alici, H. Du, Y. Zhu and W. Li, Lab Chip, 2014, 14, 2993-3003.

15. K. H. Kang, Y. Kang, X. Xuan and D. Li, Electrophoresis, 2006, 27, 694-702.

16. M. Hejazian, W. Li and N.-T. Nguyen, Lab Chip, 2015, 15, 959-970.

17. J. Zeng, C. Chen, P. Vedantam, V. Brown, T.-R. J. Tzeng and X. Xuan, J Micromech Microeng, 2012 , 22, 105018 .

18. J. S. Heyman, J Acoust Soc Am, 1993, 94, 1176-1177.

19. J. Friend and L. Y. Yeo, Rev Mod Phys., 2011, 83, 647.

20. D. J. Collins, B. L. Khoo, Z. Ma, A. Winkler, R. Weser, H. Schmidt, J. Han and Y. Ai, Lab Chip, 2017 , 17, 1769-1777.

21. M. MacDonald, G. Spalding and K. Dholakia, Nat., 2003, 426, 421-424.

22. A. Karimi, S. Yazdi and A. Ardekani, Biomicrofluidics, 2013, 7, 021501.

23. C. Liu and G. Hu, Micromachines, 2017, 8, 73.

24. N. Xiang and Z. Ni, Biomed. Microdevices, 2015, 17, 110.

25. J. Sun, M. Li, C. Liu, Y. Zhang, D. Liu, W. Liu, G. Hu and X. Jiang, Lab Chip, 2012, 12, 3952-3960.

26. M. Yamada, M. Nakashima and M. Seki, Anal. Chem., 2004, 76, 5465-5471.

27. T. A. Crowley and V. Pizziconi, Lab Chip, 2005, 5, 922-929.

28. J. Zhang, S. Yan, D. Yuan, G. Alici, N.-T. Nguyen, M. E. Warkiani and W. Li, Lab Chip, 2016, 16, 1034.

29. C. Chaffey, H. Brenner and S. Mason, Rheol Acta, 1965, 4, 64-72.

30. F. Gauthier, H. Goldsmith and S. Mason, Transactions of The Society of Rheology (1957-1977), 1971, 15, 297-330.

31. E. Bartram, H. Goldsmith and S. Mason, Rheol Acta, 1975, 14, 776-782.

32. M. Tehrani, J. Rheol., 1996, 40, 1057-1077.

33. D. F. James, Annu Rev Fluid Mech, 2009, 41, 129-142.

34. B. Ho and L. Leal, J Fluid Mech., 1976, 76, 783-799.

35. P. Huang, J. Feng, H. H. Hu and D. D. Joseph, J Fluid Mech., 1997, 343, 73-94.

36. P. Huang and D. Joseph, J Non-newtonian Fluid Mech, 2000, 90, 159-185.

37. G. D'Avino, G. Romeo, M. M. Villone, F. Greco, P. A. Netti and P. L. Maffettone, Lab Chip, 2012, 12, 1638-1645.

38. G. D'Avino, F. Greco and P. L. Maffettone, Annu Rev Fluid Mech, 2017, 49, 341-360.

39. X. Lu, C. Liu, G. Hu and X. Xuan, J Colloid Interface Sci, 2017, 500, 182-201.

40. D. Di Carlo, D. Irimia, R. G. Tompkins and M. Toner, Proc Nat Acad Sci, 2007, 104, 18892-18897.

41. J. Oakey, R. W. Applegate Jr, E. Arellano, D. D. Carlo, S. W. Graves and M. Toner, Anal. Chem., 2010, 82, 3862-3867.

42. D. Di Carlo, J. F. Edd, K. J. Humphry, H. A. Stone and M. Toner, Phys Rev Lett, 2009, 102, 094503.

43. D. Di Carlo, J. F. Edd, D. Irimia, R. G. Tompkins and M. Toner, Anal. Chem., 2008, 80, 2204-2211.

44. A. J. Mach and D. Di Carlo, Biotechnol Bioeng, 2010, 107, 302-311.

45. A. A. S. Bhagat, S. S. Kuntaegowdanahalli and I. Papautsky, Phys. Fluids, 2008, 20, 101702.

46. E. S. Asmolov, Journal of Fluid Mechanics, 1999, 381, 63-87.

47. S. Yang, J. Y. Kim, S. J. Lee, S. S. Lee and J. M. Kim, Lab Chip, 2011, 11, 266-273.

48. J. Magda, J. Lou, S. Baek and K. DeVries, Polymer, 1991, 32, 2000-2009. 
49. J. A. Pathak, D. Ross and K. B. Migler, Phys. Fluids, 2004, 16, 4028-4034.

50. M. Villone, G. D’Avino, M. Hulsen, F. Greco and P. L. Maffettone, J Non-newtonian Fluid Mech, 2011, 166, 1396-1405.

51. G. Romeo, G. D'Avino, F. Greco, P. A. Netti and P. L. Maffettone, Lab Chip, 2013, 13, 2802-2807.

52. M. Villone, G. D’Avino, M. Hulsen, F. Greco and P. L. Maffettone, J Non-newtonian Fluid Mech, 2013, 195, 1-8.

53. F. Del Giudice, G. Romeo, G. D'Avino, F. Greco, P. A. Netti and P. L. Maffettone, Lab Chip, 2013, 13, 4263-4271.

54. F. Del Giudice, G. D’Avino, F. Greco, P. A. Netti and P. L. Maffettone, Microfluid Nanofluid, 2015, 19, 95-104.

55. M. Villone, G. D’Avino, M. Hulsen, F. Greco and P. Maffettone, J Non-newtonian Fluid Mech, 2013 , 195, 1-8.

56. G. Li, G. H. McKinley and A. M. Ardekani, J Fluid Mech., 2015, 785, 486-505.

57. K. W. Seo, H. J. Byeon, H. K. Huh and S. J. Lee, RSC Adv, 2014, 4, 3512-3520.

58. K. W. Seo, Y. J. Kang and S. J. Lee, Phys. Fluids, 2014, 26, 063301.

59. D. Li, X. Lu and X. Xuan, Anal. Chem., 2016, 88, 12303-12309.

60. C. Liu, C. Xue, X. Chen, L. Shan, Y. Tian and G. Hu, Anal. Chem., 2015, 87, 6041-6048.

61. N. Xiang, Q. Dai and Z. Ni, Appl Phys Lett, 2016, 109, 134101.

62. S. H. Yang, D. J. Lee, J. R. Youn and Y. S. Song, Anal. Chem., 2017, 89, 3639-3647.

63. K. Kang, S. S. Lee, K. Hyun, S. J. Lee and J. M. Kim, Nat. Commun, 2013, 4, 2567.

64. E. J. Lim, T. J. Ober, J. F. Edd, S. P. Desai, D. Neal, K. W. Bong, P. S. Doyle, G. H. McKinley and M. Toner, Nat. Commun, 2014, 5, 4120.

65. L. Y. Yeo, H. C. Chang, P. P. Chan and J. R. Friend, small, 2011, 7, 12-48.

66. M. A. M. Ali, K. K. Ostrikov, F. A. Khalid, B. Y. Majlis and A. A. Kayani, RSC Adv, 2016, 6, 113066113094.

67. J. Nam, H. Lim, D. Kim, H. Jung and S. Shin, Lab Chip, 2012, 12, 1347-1354.

68. C. Liu, J. Guo, F. Tian, N. Yang, F. Yan, Y. Ding, J. Wei, G. Hu, G. Nie and J. Sun, ACS nano, 2017, 11 6968-6976.

69. X. Lu and X. Xuan, Anal. Chem., 2015, 87, 6389-6396.

70. J. Nam, B. Namgung, C. T. Lim, J.-E. Bae, H. L. Leo, K. S. Cho and S. Kim, J Chromatogr, 2015, 1406, 244-250.

71. D. J. Lee, H. Brenner, J. R. Youn and Y. S. Song, Sci Rep, 2013, 3, 3258.

72. D. Yuan, J. Zhang, R. Sluyter, Q. Zhao, S. Yan, G. Alici and W. Li, Lab Chip, 2016, 16, 3919-3928.

73. J. Zhang, S. Yan, D. Yuan, Q. Zhao, S. H. Tan, N.-T. Nguyen and W. Li, Lab Chip, 2016, 16, 39473956.

74. M. A. Faridi, H. Ramachandraiah, I. Banerjee, S. Ardabili, S. Zelenin and A. Russom, $J$ Nanobiotechnology, 2017, 15, 3.

75. X. Lu and X. Xuan, Anal. Chem., 2015, 87, 6389-6396.

76. J. Nam, Y. Shin, J. K. S. Tan, B. Y. Lim, C. T. Lim and S. Kim, Lab Chip, 2016, 16, 2086-2092.

77. J. Nam, J. K. S. Tan, B. L. Khoo, B. Namgung, H. L. Leo, C. T. Lim and S. Kim, Biomicrofluidics, 2015, 9, 064117.

78. H. Ramachandraiah, T. Kumar, I. Banerjee and A. Russom, 2017.

79. D. Yuan, J. Zhang, S. Yan, C. Pan, G. Alici, N.-T. Nguyen and W. Li, Biomicrofluidics, 2015, 9, 044108.

80. D. Yuan, S. H. Tan, Q. Zhao, S. Yan, R. Sluyter, N.-T. Nguyen, J. Zhang and W. Li, RSC Adv, 2017, 7, 3461-3469.

81. J. Y. Kim, S. W. Ahn, S. S. Lee and J. M. Kim, Lab Chip, 2012, 12, 2807-2814.

82. I. De Santo, G. D’Avino, G. Romeo, F. Greco, P. A. Netti and P. L. Maffettone, Phys Rev Appl., 2014, 2, 064001 .

83. C. Liu, B. Ding, C. Xue, Y. Tian, G. Hu and J. Sun, Anal. Chem., 2016, 88, 12547-12553.

84. F. Del Giudice, H. Madadi, M. M. Villone, G. D'Avino, A. M. Cusano, R. Vecchione, M. Ventre, P. L. Maffettone and P. A. Netti, Lab Chip, 2015, 15, 1912-1922.

85. M. J. Kim, D. J. Lee, J. R. Youn and Y. S. Song, RSC Adv, 2016, 6, 32090-32097.

86. S. Cha, T. Shin, S. S. Lee, W. Shim, G. Lee, S. J. Lee, Y. Kim and J. M. Kim, Anal. Chem., 2012, 84, 10471-10477.

87. K. W. Seo, Y. R. Ha and S. J. Lee, Appl Phys Lett, 2014, 104, 213702.

88. D. Yuan, S. H. Tan, R. Sluyter, Q. Zhao, S. Yan, N.-T. Nguyen, J. Guo, J. Zhang and W. Li, Anal. Chem., 2017, 89, 9574-9582.

89. D. Y. Kim, T. S. Shim and J. M. Kim, Korea Aust Rheol J., 2017, 29, 147-153.

90. S. E. Cross, Y.-S. Jin, J. Rao and J. K. Gimzewski, Nat. Nanotech., 2007, 2, 780-783. 
91. S. Suresh, Nat. Nanotech., 2007, 2, 748-749.

92. S. Chien, Annual review of physiology, 1987, 49, 177-192.

93. S. Suresh, J. Spatz, J. Mills, A. Micoulet, M. Dao, C. Lim, M. Beil and T. Seufferlein, Acta biomaterialia, 2005, 1, 15-30.

94. J. Guck, S. Schinkinger, B. Lincoln, F. Wottawah, S. Ebert, M. Romeyke, D. Lenz, H. M. Erickson, R. Ananthakrishnan and D. Mitchell, Biophys. J., 2005, 88, 3689-3698.

95. X. Mao and T. J. Huang, Lab Chip, 2012, 12, 4006-4009.

96. B. Avishay, K. Natanel, L. Alexander, L. Naomi, N. Yael and D. Uri, Rheol Acta, 2007, 46, 621-627.

97. M. M. Villone, F. Greco, M. A. Hulsen and P. L. Maffettone, J Non-newtonian Fluid Mech, 2016, 234, 105-113.

98. S. Yang, S. S. Lee, S. W. Ahn, K. Kang, W. Shim, G. Lee, K. Hyun and J. M. Kim, Soft Matter, 2012, 8, 5011-5019.

99. J. Kim, J. Y. Kim, Y. Kim, S. J. Lee and J. M. Kim, Anal. Chem., 2017, 89, 8662-8666.

100. T. Go, H. Byeon and S. J. Lee, Sci Rep, 2017, 7, 41162.

101. C.-k. Tung, C. Lin, B. Harvey, A. G. Fiore, F. Ardon, M. Wu and S. S. Suarez, Sci Rep, 2017, 7, 3152.

102. G. Holzner, S. Stavrakis and A. J. deMello, Anal. Chem., 2017, 89, 11653-11663.

103. K. Jayaprakash, U. Banerjee and A. Sen, Langmuir, 2016, 32, 2136-2143.

104. K. Jayaprakash, U. Banerjee and A. Sen, J Colloid Interface Sci, 2017, 493, 317-326.

105. D. Yuan, J. Zhang, S. Yan, G. Peng, Q. Zhao, G. Alici, H. Du and W. Li, ELECTROPHORESIS, 2016, 37, 2147-2155.

106. B. Ha, J. Park, G. Destgeer, J. H. Jung and H. J. Sung, Anal. Chem., 2016, 88, 4205-4210.

107. F. Tian, W. Zhang, L. Cai, S. Li, G. Hu, Y. Cong, C. Liu, T. Li and J. Sun, Lab Chip, 2017, 17, 30783085.

108. S. Gupta, W. S. Wang and S. A. Vanapalli, Biomicrofluidics, 2016, 10, 043402.

109. S. Haward, Biomicrofluidics, 2016, 10, 043401.

110. K.-W. Hsiao, J. Dinic, Y. Ren, V. Sharma and C. M. Schroeder, Phys. Fluids, 2017, 29, 121603.

111. A. E. Koser, L. Pan, N. C. Keim and P. E. Arratia, Lab Chip, 2013, 13, 1850-1853.

112. J. Zilz, C. Schäfer, C. Wagner, R. J. Poole, M. A. Alves and A. Lindner, Lab Chip, 2014, 14, 351-358.

113. F. Del Giudice, G. D'Avino, F. Greco, I. De Santo, P. A. Netti and P. L. Maffettone, Lab Chip, 2015, 15, 783-792.

114. F. Del Giudice, V. Calcagno, V. Esposito Taliento, F. Greco, P. A. Netti and P. L. Maffettone, Journal of Rheology, 2017, 61, 13-21.

115. F. Del Giudice, S. J. Haward and A. Q. Shen, Journal of Rheology, 2017, 61, 327-337.

116. S. O. Hong, J. J. Cooper-White and J. M. Kim, Appl Phys Lett, 2016, 108, 014103.

117. L. A. N. Julius, V. K. Jagannadh, I. J. Michael, R. Srinivasan and S. S. Gorthi, BioChip Journal, 2016, 10, 16-24.

118. K. P. Nolan, A. Agarwal, S. Lei and E. Dalton, 2016.

119. D.-Y. Li, X.-B. Li, H.-N. Zhang, F.-C. Li, S.-Z. Qian and S. W. Joo, Exp Therm Fluid Sci, 2016, 77, 226-233.

120. A. C. Hatch, A. Patel, N. R. Beer and A. P. Lee, Lab Chip, 2013, 13, 1308-1315.

121. W. Lee, H. Amini, H. A. Stone and D. Di Carlo, Proc Nat Acad Sci, 2010, 107, 22413-22418.

122. S. Kahkeshani, H. Haddadi and D. Di Carlo, J Fluid Mech., 2016, 786.

123. K. J. Humphry, P. M. Kulkarni, D. A. Weitz, J. F. Morris and H. A. Stone, Phys. Fluids, 2010, 22, 081703 .

124. A. E. Reece and J. Oakey, Phys. Fluids, 2016, 28, 043303.

125. M. Tehrani, Journal of Rheology, 1996, 40, 1057-1077.

126. A. W. Richter and E. Åkerblom, Int Arch Allergy Immunol, 1983, 70, 124-131.

127. J. D. Glass, L. Silver, J. Sondheimer, C. S. Pande and J. Coderre, Biopolymers, 1979, 18, 383-392.

128. K. Ulbrich, J. Strohalm and J. Kopeček, Macromol Chem Phys., 1986, 187, 1131-1144.

129. J. M. Harris, E. C. Struck, M. G. Case, M. S. Paley, M. Yalpani, J. M. Van Alstine and D. E. Brooks, J Polym Sci A Polym Chem., 1984, 22, 341-352.

130. A. Abuchowski, T. Van Es, N. Palczuk and F. Davis, J. Biol. Chem., 1977, 252, 3578-3581.

131. Y. Ashihara, T. Kono, S. Yamazaki and Y. Inada, Biochem Biophys Res Commun., 1978, 83, 385-391. 


\section{Graphical abstract}

In this review, we discuss the up-to-date progress of particle migration in viscoelastic fluids mainly from the aspect of applications, laying out a comprehensive perspective on their potentials in future lab-on-a-chip platforms.

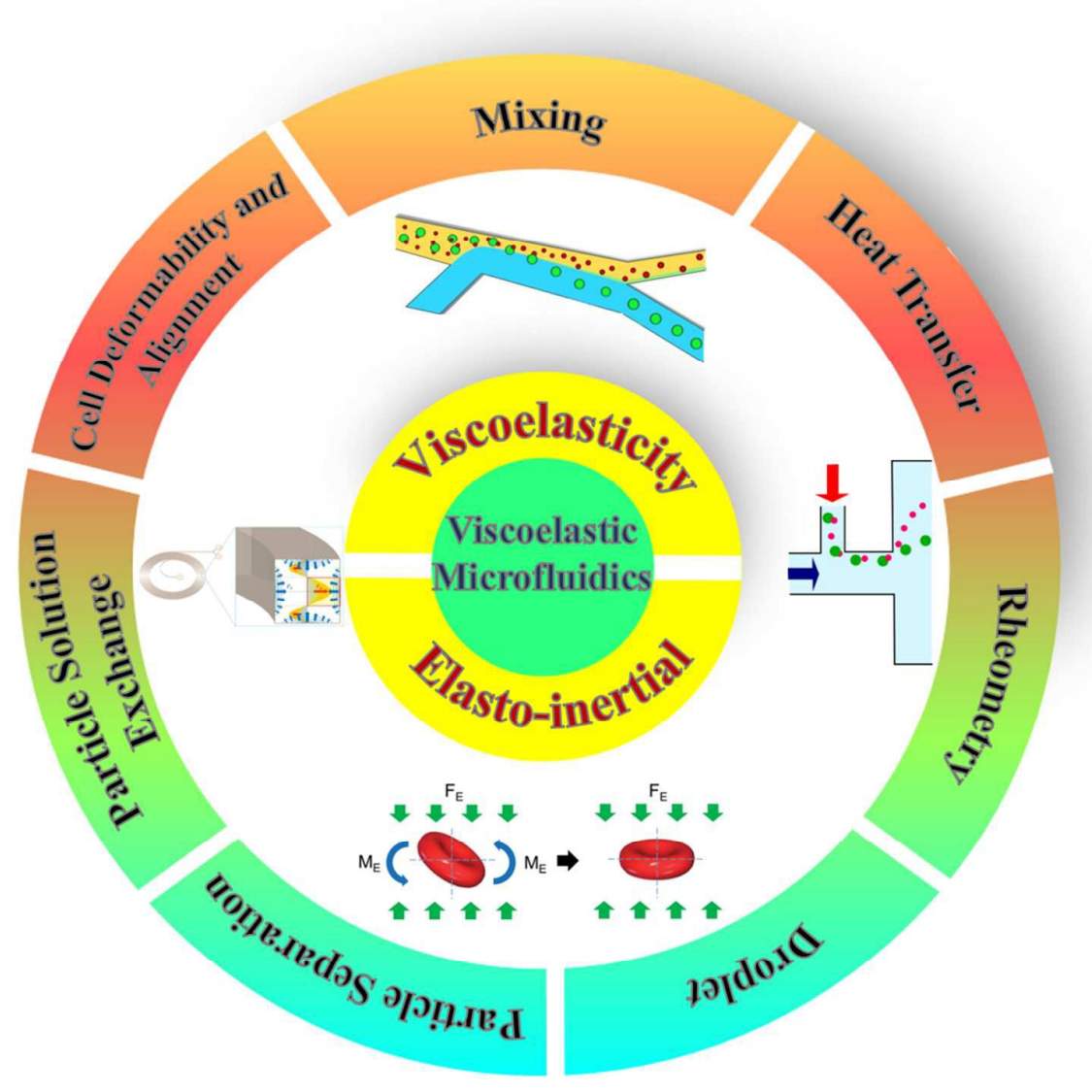

Figure 1 Overview of particle migration in viscoelastic fluids. 


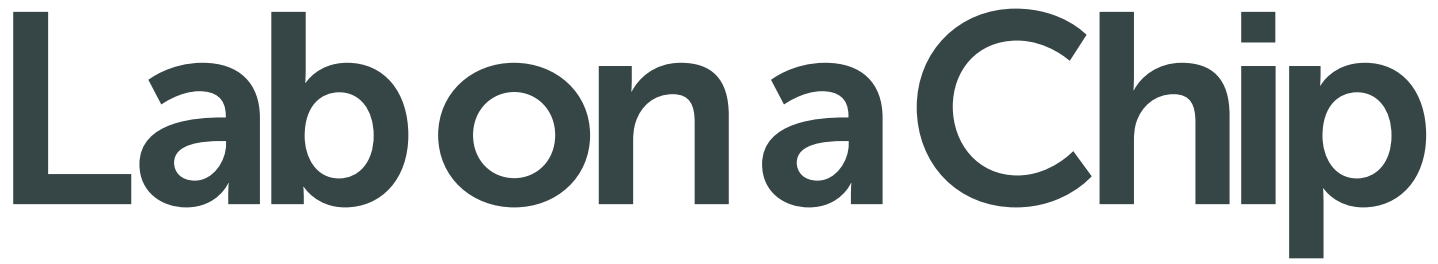

\section{Accepted Manuscript}

This article can be cited before page numbers have been issued, to do this please use: D. Yuan, Q. Zhao, S. Yan, S. Tang, G. ALICI, J. Zhang and W. Li, Lab Chip, 2018, DOI: 10.1039/C7LC01076A.

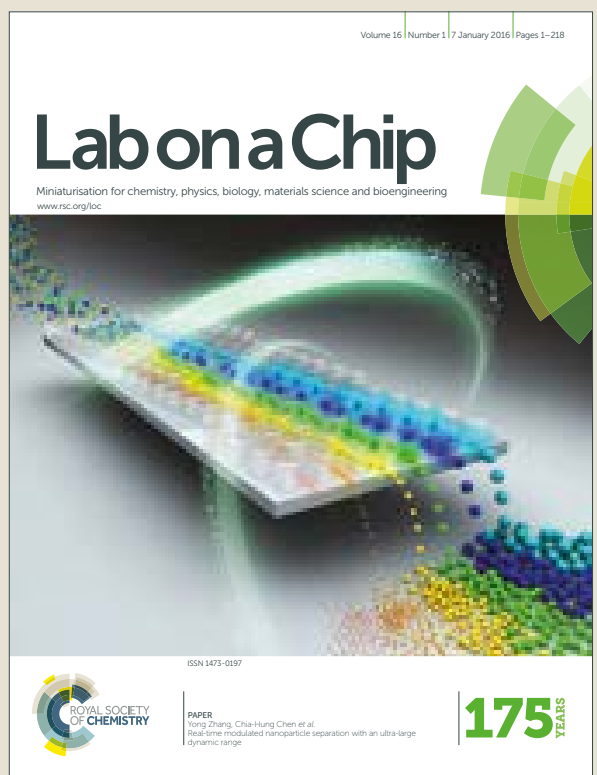

This is an Accepted Manuscript, which has been through the Royal Society of Chemistry peer review process and has been accepted for publication.

Accepted Manuscripts are published online shortly after acceptance, before technical editing, formatting and proof reading. Using this free service, authors can make their results available to the community, in citable form, before we publish the edited article. We will replace this Accepted Manuscript with the edited and formatted Advance Article as soon as it is available.

You can find more information about Accepted Manuscripts in the author guidelines.

Please note that technical editing may introduce minor changes to the text and/or graphics, which may alter content. The journal's standard Terms \& Conditions and the ethical guidelines, outlined in our author and reviewer resource centre, still apply. In no event shall the Royal Society of Chemistry be held responsible for any errors or omissions in this Accepted Manuscript or any consequences arising from the use of any information it contains. 